TRANSACTIONS OF THE

AMERICAN MATHEMATICAL SOCIETY

Volume 359, Number 10, October 2007, Pages 4595-4623

S 0002-9947(07)03850-0

Article electronically published on May 11, 2007

\title{
RECURSIVE SUBHOMOGENEOUS ALGEBRAS
}

\author{
N. CHRISTOPHER PHILLIPS
}

\begin{abstract}
We introduce and characterize a particularly tractable class of unital type $1 \mathrm{C}^{*}$-algebras with bounded dimension of irreducible representations. Algebras in this class are called recursive subhomogeneous algebras, and they have an inductive description (through iterated pullbacks) which allows one to carry over from algebras of the form $C\left(X, M_{n}\right)$ many of the constructions relevant in the study of the stable rank and K-theory of simple direct limits of homogeneous $\mathrm{C}^{*}$-algebras. Our characterization implies, in particular, that if $A$ is a separable $C^{*}$-algebra whose irreducible representations all have dimension at most $N<\infty$, and if for each $n$ the space of $n$-dimensional irreducible representations has finite covering dimension, then $A$ is a recursive subhomogeneous algebra. We demonstrate the good properties of this class by proving subprojection and cancellation theorems in it.

Consequences for simple direct limits of recursive subhomogeneous algebras, with applications to the transformation group $\mathrm{C}^{*}$-algebras of minimal homeomorphisms, will be given in separate papers.
\end{abstract}

\section{IntroduCtion}

In recent years, a number of results have been proved about the stable and real rank and the unstable K-theory of certain kinds of direct limits of homogeneous $\mathrm{C}^{*}$-algebras, usually assuming some kind of slow dimension growth and sometimes assuming simplicity. See for example [8], [5], 3], 26], and [15. The subprojection and cancellation theorems for algebras of the form $C\left(X, M_{n}\right)$ are important ingredients in many of the proofs. These theorems essentially say that if $\operatorname{rank}(p)-\operatorname{rank}(q)$ is large enough compared to the dimension of $X$, then $q$ is a subprojection of $p$, and that if $\operatorname{rank}(p)$ is large enough compared to the dimension of $X$, and if $p \oplus e$ is Murray-von Neumann equivalent to $q \oplus e$, then $p$ is Murray-von Neumann equivalent to $q$. See Chapter 9 of [16] for the original formulation in terms of vector bundles over finite complexes, and see Theorem 2.5 of [15], Lemma 3.4 of [26], and Lemma 1.5 of 34 for $\mathrm{C}^{*}$-algebraic formulations.

In this paper, we introduce a class of type $1 \mathrm{C}^{*}$-algebras, much more general than those of the form $C\left(X, M_{n}\right)$, in which analogs of these theorems are still true. Algebras in this class, called recursive subhomogeneous algebras, can be expressed as iterated pullbacks of algebras of the form $C\left(X, M_{n}\right)$, in such a way that an inductive argument reduces these theorems to "relative" versions of the same theorems for $C\left(X, M_{n}\right)$. In two further papers, [35] and [36], we use these results to generalize some of the known results on direct limits of direct sums of homogeneous

Received by the editors January 22, 2001 and, in revised form, August 2, 2004.

2000 Mathematics Subject Classification. Primary 46L05; Secondary 19A13, 19B14, 19K14, $46 \mathrm{~L} 80$.

This research was partially supported by NSF grants DMS 9400904 and DMS 9706850. 
$\mathrm{C}^{*}$-algebras to direct limits of recursive subhomogeneous algebras. Combining these results on direct limits with the work of Qing Lin [21, we will obtain information on the order on the $K_{0}$ groups of $\mathrm{C}^{*}$-algebras of minimal homeomorphisms. Moreover, recursive subhomogeneous algebras play a crucial role in recent work on the structure and classification of $\mathrm{C}^{*}$-algebras of minimal homeomorphisms of spaces of dimension greater than one [23, [24, 20].

Recursive subhomogeneous algebras include a number of algebras which have already played significant roles in the study of direct limits of type $1 \mathrm{C}^{*}$-algebras, and also elsewhere. The following are all recursive subhomogeneous algebras:

- Finite direct sums of algebras of the form $C\left(X, M_{n}\right)$ for $X$ compact Hausdorff and $n \geq 1$.

- Dimension drop intervals (as used in [12]) and matrix algebras over them.

- The building blocks used in the classification theorems of [17, [18, [42, [29], and [30].

- The noncommutative CW complexes of 32 .

- Section algebras of locally trivial continuous fields over $X$ with fiber $M_{n}$ (with arbitrary Dixmier-Douady class).

- The algebras $A_{Y}$ arising in Qing Lin's study [21] of the transformation group $\mathrm{C}^{*}$-algebras of minimal homeomorphisms, provided $\operatorname{int}(Y) \neq \varnothing$. (See Example 1.6 for more details.)

It is obvious from the definition (given in Section 1) that recursive subhomogeneous algebras are unital type $1 \mathrm{C}^{*}$-algebras with a finite upper bound on the possible dimensions of irreducible representations. Among such algebras, they at first seem rather special, but in fact there are a great many recursive subhomogeneous algebras. We prove in Section 2 a characterization theorem which implies the following: If $A$ is a separable $\mathrm{C}^{*}$-algebra whose irreducible representations all have dimension at most $N<\infty$, and if for each $n$ the space of $n$-dimensional irreducible representations has finite covering dimension, then $A$ is a recursive subhomogeneous algebra.

Work related to our first two sections has been done in [44]. (We are grateful to George Elliott for pointing out this reference.) Specifically, Theorem 4 of 44 . gives a decomposition of an arbitrary subhomogeneous $\mathrm{C}^{*}$-algebra which is related to that in our Proposition 2.13. (Note that we show the decomposition described in Proposition 2.13 holds under the hypotheses of Theorems 2.15 and 2.16]) However, the description of 44 is much more complicated and difficult to work with. In our applications (see, for example, [35] and [36]), the spaces of irreducible representations of each fixed dimension will all have finite covering dimension, in which case Theorem 2.16 guarantees the applicability of Proposition 2.13. Moreover, in the work outlined in Section 6 of [22], we apparently need recursive subhomogeneous decompositions satisfying an additional condition (a suitable a priori bound on the "strong covering number"). As far as we know, the decomposition of 44 need not satisfy any analog of this condition; nor do we know how to obtain it for the decomposition of Proposition 2.13 .

There are four sections. The first section contains the definition of a recursive subhomogeneous algebra, examples, elementary closure properties of the class, and related results. The second gives a characterization of separable recursive subhomogeneous algebras, both in general and under the condition that the spaces of $n$-dimensional irreducible representations have finite covering dimension. In the 
third section, these results are used to give stronger closure properties of the class of recursive subhomogeneous algebras. In particular, quotients, unitized ideals, suitable pullbacks, and tensor products of recursive subhomogeneous algebras are all again recursive subhomogeneous algebras. However, it is shown by example that subalgebras of recursive subhomogeneous algebras need not be recursive subhomogeneous algebras. In Section 4 we prove the subprojection and cancellation theorems for recursive subhomogeneous algebras, and an analogous result about unitaries. Applying the results of the previous sections, we obtain subprojection and cancellation theorems for separable $\mathrm{C}^{*}$-algebras with a finite upper bound on the possible dimensions of irreducible representations, under suitable assumptions on the dimensions of subspaces of the primitive ideal space. We also give a sufficient condition for the $K_{1}$-group to be determined by the unitaries in the algebra, without using matrices.

In this paper, we let $\operatorname{dim}(X)$ denote the modified covering dimension of the topological space $X$. See Definition 10.1.3 of [31]. This is the same as the usual covering dimension (Definition 3.1.1 of 31]) on compact Hausdorff spaces. Since we only use other spaces in Section 2, we postpone further discussion to there. (Warning on terminology: "bicompact" in 31 is what is usually called compact Hausdorff, and "Tihonov" in 31 is what is usually called completely regular, that is, points are closed and can be separated from closed sets by continuous functions.)

I am grateful to Larry Brown, Maurice Dupré, Qing Lin, Gert Pedersen, and Claude Schochet for useful conversations and email correspondence. In particular, the definition of a recursive subhomogeneous algebra arose from an effort with Qing Lin to impose structure on the algebras arising in his study 21] of the transformation group $\mathrm{C}^{*}$-algebras of minimal homeomorphisms (see Example 1.6), and Larry Brown suggested the possible relevance of the finite type condition used in Section 2. This work was carried out during a sabbatical year at Purdue University, and I am grateful to that institution for its hospitality.

Some of the results of this paper were announced in $[22]$.

\section{RECURSive SUbHomogeneous ALGEBras}

In this section, we give the definition of a recursive subhomogeneous algebra, along with several remarks and some useful terminology. We then give a number of examples, including all those mentioned in the introduction. After that, we give some closure properties of the class of recursive subhomogeneous algebras which can be proved easily from the definition. In particular, direct sums and corners of recursive subhomogeneous algebras are recursive subhomogeneous algebras, and tensor products of recursive subhomogeneous algebras with $C(X)$ and $M_{n}$ are recursive subhomogeneous algebras. Some stronger closure properties will be obtained in Section 3 .

Definition 1.1. The class of recursive subhomogeneous algebras is the smallest class $\mathcal{R}$ of $\mathrm{C}^{*}$-algebras which is closed under isomorphism and such that:

(1) If $X$ is a compact Hausdorff space and $n \geq 1$, then $C\left(X, M_{n}\right) \in \mathcal{R}$.

(2) $\mathcal{R}$ is closed under the following pullback construction: If $A \in \mathcal{R}$, if $X$ is a compact Hausdorff space, if $X^{(0)} \subset X$ is closed, if $\varphi: A \rightarrow C\left(X^{(0)}, M_{n}\right)$ is any unital homomorphism, and if $\rho: C\left(X, M_{n}\right) \rightarrow C\left(X^{(0)}, M_{n}\right)$ is the 
restriction homomorphism, then the pullback

$$
A \oplus_{C\left(X^{(0)}, M_{n}\right)} C\left(X, M_{n}\right)=\left\{(a, f) \in A \oplus C\left(X, M_{n}\right): \varphi(a)=\rho(f)\right\}
$$

is in $\mathcal{R}$ (compare with Definition 2.1 of [32]).

Note that in (2) the choice $X^{(0)}=\varnothing$ is allowed (in which case $\varphi=0$ is allowed). Thus the pullback could be an ordinary direct sum.

It is convenient in several situations (such as consideration of corners) to allow the zero algebra to be a recursive subhomogeneous algebra.

Examples will be presented shortly, but some terminology and a warning are appropriate first.

Definition 1.2. We adopt the following standard notation for recursive subhomogeneous algebras. From the definition, it is clear that any recursive subhomogeneous algebra can be written in the form

$$
R \cong\left[\cdots\left[\left[C_{0} \oplus_{C_{1}^{(0)}} C_{1}\right] \oplus_{C_{2}^{(0)}} C_{2}\right] \cdots\right] \oplus_{C_{l}^{(0)}} C_{l},
$$

with $C_{k}=C\left(X_{k}, M_{n(k)}\right)$ for compact Hausdorff spaces $X_{k}$ and positive integers $n(k)$, with $C_{k}^{(0)}=C\left(X_{k}^{(0)}, M_{n(k)}\right)$ for compact subsets $X_{k}^{(0)} \subset X_{k}$ (possibly empty), and where the maps $C_{k} \rightarrow C_{k}^{(0)}$ are always the restriction maps. An expression of this type will be referred to as a decomposition of $R$, and the notation used here will be referred to as the standard notation for a decomposition.

Associated with this decomposition are:

(1) its length l;

(2) the $k$-th stage algebra

$$
R^{(k)}=\left[\cdots\left[\left[C_{0} \oplus_{C_{1}^{(0)}} C_{1}\right] \oplus_{C_{2}^{(0)}} C_{2}\right] \cdots\right] \oplus_{C_{k}^{(0)}} C_{k},
$$

obtained by using only the first $k+1$ algebras $C_{0}, C_{1}, \ldots, C_{k}$;

(3) its base spaces $X_{0}, X_{1}, \ldots, X_{l}$ and total space $X=\coprod_{k=0}^{l} X_{k}$;

(4) its matrix sizes $n(0), \ldots, n(l)$, and matrix size function $m: X \rightarrow \mathbf{N} \cup\{0\}$, defined by $m(x)=n(k)$ when $x \in X_{k}$ (this is called the matrix size of $A$ at $x)$;

(5) its minimum matrix size $\min _{k} n(k)$ and maximum matrix size $\max _{k} n(k)$;

(6) its topological dimension $\operatorname{dim}(X)$ (the covering dimension of $X$ 31, Definition 3.1.1; here equal to $\max _{k} \operatorname{dim}\left(X_{k}\right)$ ), and topological dimension function $d: X \rightarrow \mathbf{N} \cup\{0\}$, defined by $d(x)=\operatorname{dim}\left(X_{k}\right)$ when $x \in X_{k}$ (this is called the topological dimension of $A$ at $x)$;

(7) its standard representation $\sigma=\sigma_{R}: R \rightarrow \bigoplus_{k=0}^{l} C\left(X_{k}, M_{n(k)}\right)$, defined by forgetting the restriction to a subalgebra in each of the fibered products in the decomposition;

(8) the associated evaluation maps $\mathrm{ev}_{x}: R \rightarrow M_{n(k)}$ for $x \in X_{k}$, defined to be the restriction of the usual evaluation map to $R$, identified with a subalgebra of $\bigoplus_{k=0}^{l} C\left(X_{k}, M_{n(k)}\right)$ via $\sigma$.

Warning 1.3. The decomposition of a recursive subhomogeneous algebra is highly nonunique (as will become clear from some of the examples). Throughout this paper, we will tacitly assume (unless otherwise specified) that every recursive subhomogeneous algebra is given with some decomposition. In particular, we will refer 
to the length, matrix sizes, etc. of a recursive subhomogeneous algebra, by which we mean the corresponding quantities for a tacitly understood given decomposition of the algebra.

We now give examples; for later use, some are given as parts of propositions.

Example 1.4. Any finite direct sum of $\mathrm{C}^{*}$-algebras of the form $C\left(X, M_{n}\right)$ (trivial homogeneous algebras) is a recursive subhomogeneous algebra. More generally, any finite direct sum of recursive subhomogeneous algebras is a recursive subhomogeneous algebra.

Example 1.5. The noncommutative CW complexes of Section 11 of 32 are recursive subhomogeneous algebras (with particularly nice base spaces) whenever they are unital. In particular, the "dimension drop intervals"

$$
\widetilde{I}_{n}=\left\{f \in C\left([0,1], M_{n}\right): f(0), f(1) \in \mathbf{C} \cdot 1\right\}
$$

and the matrix algebras $M_{k}\left(\widetilde{I}_{n}\right)$ are recursive subhomogeneous algebras. (These were introduced in [6], and have since played a significant role in the stably finite classification theory; see, for example, [12] and [7.)

To write $\widetilde{I}_{n}$ as a recursive subhomogeneous algebra of length 1 , set

$$
C_{0}=\mathbf{C} \oplus \mathbf{C}, \quad C_{1}=C\left([0,1], M_{n}\right), \quad \text { and } \quad C_{1}^{(0)}=M_{n} \oplus M_{n},
$$

and define maps from $C_{0}$ and $C_{1}$ to $C_{1}^{(0)}$ by $\varphi_{1}(\alpha, \beta)=(\alpha \cdot 1, \beta \cdot 1)$ and $\rho_{1}(f)=$ $(f(0), f(1))$. To get $M_{k}\left(\widetilde{I}_{n}\right)$, form $k \times k$ matrices over everything. (Compare with Lemma 1.12 below.)

Algebras which are generalizations of the dimension drop intervals, but are still one-dimensional noncommutative CW complexes, have been used as building blocks in classification theorems for simple direct limits in [17, 18], 42], 29], and [30].

Example 1.6. Let $X$ be an infinite compact metric space, let $h$ be a minimal homeomorphism of $X$, and let $Y \subset X$ be closed. Let $A=C^{*}(\mathbf{Z}, X, h)$ be the transformation group $C^{*}$-algebra, with unitary $u$ representing the generator of $\mathbf{Z}$. Let

$$
A_{Y}=C^{*}\left(C(X), u C_{0}(X \backslash Y)\right) \subset C^{*}(\mathbf{Z}, X, h) .
$$

The computations in the proof of Theorem 3 of 21] (also see Section 3 of [22]) show that, if $\operatorname{int}(Y) \neq \varnothing$, then $A_{Y}$ is a recursive subhomogeneous algebra, with topological dimension at most $\operatorname{dim}(X)$. (This uses the fact that if $Z \subset X$ is closed, then $\operatorname{dim}(Z) \leq \operatorname{dim}(X)$. See Proposition 3.1.5 of 31.)

It follows immediately that if $y_{0} \in X$, then $A_{\left\{y_{0}\right\}}$ is a direct limit of recursive subhomogeneous algebras, with all maps in the direct system injective and unital. In fact, if

$$
Y_{1} \supset Y_{2} \supset \cdots, \quad \operatorname{int}\left(Y_{n}\right) \neq \varnothing, \quad \text { and } \bigcap_{n=1}^{\infty} Y_{n}=\left\{y_{0}\right\},
$$

then $A_{\left\{y_{0}\right\}}=\overline{\bigcup_{n=1}^{\infty} A_{Y_{n}}}$.

We next show that locally trivial continuous fields are recursive subhomogeneous algebras. This follows from the following more general result, which has other useful consequences. 
Proposition 1.7. Let $X$ be a compact Hausdorff space, let $E$ be a locally trivial continuous field over $X$ with fiber $M_{n}$, and let $B=\Gamma(E)$ be the corresponding section algebra. (We make no restriction on the Dixmier-Douady class.) Let $A$ be a $C^{*}$-algebra with a recursive subhomogeneous decomposition of length $l_{0}$, topological dimension $d_{0}$, and maximum matrix size $n_{0}$. Let $\varphi: A \rightarrow C$ and $\rho: B \rightarrow C$ be unital homomorphisms, with $\rho$ surjective. (As usual, we allow the case $C=0, \varphi=0$, and $\rho=0$.) Then there exists a recursive subhomogeneous decomposition for $A \oplus_{C} B$ such that, adopting the standard notation (from Definition 1.2), the following hold:

(1) The $l_{0}$-th stage algebra (Definition 1.2) of this recursive subhomogeneous decomposition is $A$, and its obvious recursive subhomogeneous decomposition of length $l_{0}$ is the given recursive subhomogeneous decomposition of A.

(2) For $l_{0}<k \leq l$, we have $\operatorname{dim}\left(X_{k}\right) \leq \operatorname{dim}(X)$.

(3) For $l_{0}<k \leq l$, the matrix size $n(k)$ is equal to $n$.

In particular, $A \oplus_{C} B$ has a recursive subhomogeneous decomposition whose topological dimension is $\max \left(d_{0}, \operatorname{dim}(X)\right)$, and whose maximum matrix size is $\max \left(n_{0}, n\right)$.

Moreover, if $X^{(0)} \subset X$ is the closed subset of $X$ such that

$$
\operatorname{Ker}(\rho)=\left\{f \in \Gamma(E):\left.f\right|_{X^{(0)}}=0\right\},
$$

and if $X \backslash X^{(0)}$ is dense in $X$, then the recursive subhomogeneous decomposition may be chosen to have the additional property:

(4) For $l_{0}<k \leq l$, we have $X_{k} \backslash X_{k}^{(0)}$ dense in $X_{k}$.

Proof. We may clearly assume $C=\Gamma\left(\left.E\right|_{X^{(0)}}\right)$ with $X^{(0)} \subset X$ closed, and that $\rho$ is the restriction map. Using compactness of $X$ and local triviality, cover $X$ with finitely many open sets $U_{1}, \ldots, U_{r}$ such that $\left.E\right|_{\bar{U}_{j}}$ is trivial for $1 \leq j \leq r$. We prove the proposition by induction on $r$. For $r=1$, we have $B \cong C\left(X, M_{n}\right)$, and $A \oplus_{C} B$ has an obvious recursive subhomogeneous decomposition of length $l=l_{0}+1$. Moreover, the properties (1), (2), and (3) (and (4), if applicable) are immediate.

So suppose the proposition is known in all cases when $r$ sets $U_{1}, \ldots, U_{r}$ suffice, and suppose $E$ is given, with a cover $\left\{U_{1}, \ldots, U_{r+1}\right\}$ such that $\left.E\right|_{\bar{U}_{j}}$ is trivial for $1 \leq j \leq r+1$. Define

$$
\widetilde{X}=\bigcup_{j=1}^{r} \bar{U}_{j}, \quad \widetilde{X}^{(0)}=\widetilde{X} \cap X^{(0)}, \quad \widetilde{B}=\Gamma\left(\left.E\right|_{\widetilde{X}}\right), \quad \text { and } \quad \widetilde{C}=\Gamma\left(\left.E\right|_{\widetilde{X}^{(0)}}\right) .
$$

Let $\pi: C \rightarrow \widetilde{C}$ be the restriction map. Let $\widetilde{\varphi}=\pi \circ \varphi: A \rightarrow \widetilde{C}$, and let $\widetilde{\rho}: \widetilde{B} \rightarrow \widetilde{C}$ be given by restriction of sections from $\widetilde{X}$ to $\widetilde{X}^{(0)}$. Then $\widetilde{\varphi}$ and $\widetilde{\rho}$ are unital, and $\widetilde{\rho}$ is surjective. By the induction hypothesis, $A \oplus_{\widetilde{C}} \widetilde{B}$ has a recursive subhomogeneous algebra such that the properties (1), (2), and (3) hold.

Define

$$
Y=\overline{X \backslash \widetilde{X}} \text { and } Y^{(0)}=Y \cap\left(\widetilde{X} \cup X^{(0)}\right) .
$$

Then $Y$ and $\tilde{X}$ are closed subsets of $X$ which cover $X$. Without loss of generality, $Y \neq \varnothing$. Now set

$$
B_{0}=\Gamma\left(\left.E\right|_{Y}\right) \quad \text { and } \quad C_{0}=\Gamma\left(\left.E\right|_{Y^{(0)}}\right) .
$$

Note that $B_{0} \cong C\left(Y, M_{n}\right)$ because $Y \subset \bar{U}_{r+1}$ and $\left.E\right|_{\bar{U}_{r+1}}$ is trivial. Let $\rho_{0}: B_{0} \rightarrow$ $C_{0}$ be the restriction map. Define $\varphi_{0}: A \oplus_{\widetilde{C}} \widetilde{B} \rightarrow C_{0}$ as follows. For $(a, f) \in A_{\oplus_{\widetilde{C}}} \widetilde{B}$, 
we let $\varphi_{0}(a, f)$ be the section in $C_{0}$ given by

$$
\left.\varphi_{0}(a, f)\right|_{Y \cap \widetilde{X}}=\left.f\right|_{Y \cap \widetilde{X}} \quad \text { and }\left.\quad \varphi_{0}(a, f)\right|_{Y \cap X^{(0)}}=\left.\varphi(a)\right|_{Y \cap X^{(0)}} .
$$

The condition that $(a, f)$ be in $A \oplus_{\widetilde{C}} \widetilde{B}$ ensures that we do in fact have

$$
\left.f\right|_{Y \cap \widetilde{X} \cap X^{(0)}}=\left.\varphi(a)\right|_{Y \cap \widetilde{X} \cap X^{(0)}} .
$$

We now claim that

$$
A \oplus_{C} B \cong\left(A \oplus_{\widetilde{C}} \widetilde{B}\right) \oplus_{C_{0}} B_{0} .
$$

To see this, simply observe that the right-hand side is the set of all triples

$$
(a, f, g) \in A \oplus \widetilde{B} \oplus B_{0}
$$

such that

$$
\left.\varphi(a)\right|_{\widetilde{X}^{(0)}}=\left.f\right|_{\widetilde{X}^{(0)}},\left.\quad \varphi(a)\right|_{Y \cap X^{(0)}}=\left.g\right|_{Y \cap X^{(0)}}, \quad \text { and }\left.\quad f\right|_{Y \cap \widetilde{X}}=\left.g\right|_{Y \cap \widetilde{X}} .
$$

The map that sends $(a, h) \in A \oplus_{C} B$ to $\left(a,\left.h\right|_{\widetilde{X}},\left.h\right|_{Y}\right)$ is now obviously an isomorphism.

The $\mathrm{C}^{*}$-algebra $\left(A \oplus_{\widetilde{C}} \widetilde{B}\right) \oplus_{C_{0}} B_{0}$ has an obvious recursive subhomogeneous decomposition, with length 1 larger than that for $A \oplus_{\widetilde{C}} \widetilde{B}$. Moreover, the conditions (1), (2), and (3) clearly hold (using Proposition 3.1.5 of [31] for the dimension estimate in (2)).

It remains to show that if $X \backslash X^{(0)}$ is dense in $X$, then condition (4) also holds. By induction, we need only show that $\widetilde{X} \backslash \widetilde{X}^{(0)}$ is dense in $\widetilde{X}$ and $Y \backslash Y^{(0)}$ is dense in $Y$. For the first of these, it suffices to show that each $U_{j} \cap\left(X \backslash X^{(0)}\right)$ is dense in $U_{j}$, since this set is then necessarily dense in $\bar{U}_{j}$. This is easy because $U_{j}$ is open and $X \backslash X^{(0)}$ is dense in $X$. For the second, it suffices to prove that $(X \backslash \widetilde{X}) \backslash Y^{(0)}$ is dense in $X \backslash \widetilde{X}$. This is true because

$$
(X \backslash \tilde{X}) \backslash Y^{(0)}=(X \backslash \widetilde{X}) \cap\left(X \backslash X^{(0)}\right),
$$

and $X \backslash \widetilde{X}$ is open and $X \backslash X^{(0)}$ is dense.

Corollary 1.8. Let $X$ be a compact Hausdorff space, let $E$ be a locally trivial continuous field over $X$ with fiber $M_{n}$, and let $A=\Gamma(E)$ be the corresponding section algebra. Then $A$ is a recursive subhomogeneous algebra.

In Section 3, we prove, in the separable case and using more machinery, that $A \oplus_{C} B$ is a recursive subhomogeneous algebra when $B$ is merely required to be a recursive subhomogeneous algebra.

To obtain the other interesting corollaries, the following lemma is useful.

Lemma 1.9. Let $A, B_{1}, B_{2}$, and $C$ be unital $C^{*}$-algebras (allowing, however, $C=0$ ), and let $\varphi: A \rightarrow C$ and $\rho: B_{1} \oplus B_{2} \rightarrow C$ be unital homomorphisms, with $\rho$ surjective. Then there are unital $C^{*}$-algebras $C_{1}$ and $C_{2}$ (possibly the zero $C^{*}$ algebra) and unital homomorphisms

$$
\varphi_{1}: A \rightarrow C_{1}, \quad \rho_{1}: B_{1} \rightarrow C_{1}, \quad \varphi_{2}: A \oplus_{C_{1}} B_{1} \rightarrow C_{2}, \quad \text { and } \rho_{2}: B_{2} \rightarrow C_{2},
$$

with $\rho_{1}$ and $\rho_{2}$ surjective, such that $A \oplus_{C}\left(B_{1} \oplus B_{2}\right) \cong\left(A \oplus_{C_{1}} B_{1}\right) \oplus_{C_{2}} B_{2}$.

Proof. We have $C=C_{1} \oplus C_{2}$, where $C_{j}=\rho\left(B_{j}\right)$. Let $\rho_{j}: B_{j} \rightarrow C_{j}$ be the corresponding restriction of $\rho$. Let $\pi_{j}: C \rightarrow C_{j}$ be the projection map, and define $\varphi_{1}=\pi_{1} \circ \varphi$ and $\varphi_{2}(a, b)=\pi_{2} \circ \varphi(a)$. It is easy to check that the map $\left(a, b_{1}, b_{2}\right) \mapsto\left(a, b_{1}, b_{2}\right)$ is an isomorphism of the two different fibered products. 
Corollary 1.10. In Definition 1.1 we may replace $C\left(X, M_{n}\right)$ by an arbitrary locally trivial homogeneous algebra without changing the class of algebras defined. Moreover, given a decomposition of this weaker kind, there is a true decomposition with the same topological dimension and set of matrix sizes.

Proof. We use Lemma 1.9 to reduce to the case of constant rank, which is then covered by induction and Proposition 1.7

Corollary 1.11. Let $A$ be a recursive subhomogeneous algebra, and let $p \in A$ be a projection. Then $p A p$ is a recursive subhomogeneous algebra, with topological dimension and maximum matrix size no larger than for $A$.

Proof. Let $A$ have a decomposition in the standard notation (as in Definition 1.2). Let $p_{k}$ be the image of $p$ in $C_{k}$, and let $p_{k}^{(0)}$ be the image of $p_{k}$ in $C_{k}^{(0)}$. Then

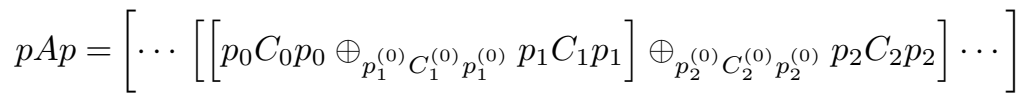

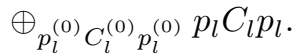

Omitting terms for which $p_{k}=0$, the conclusion follows from Corollary 1.10 ,

Lemma 1.12. Let $A$ be a recursive subhomogeneous algebra. Then $M_{n}(A)$ is a recursive subhomogeneous algebra. It has a decomposition with the same length and base spaces as for $A$, and with maximum matrix size equal to $n$ times the maximum matrix size of $A$.

Proof. This is immediate from the definition.

Corollary 1.13. Let $A$ be a recursive subhomogeneous algebra. Let $B$ be a unital $C^{*}$-algebra which is Morita equivalent to $A$. Then $B$ is a recursive subhomogeneous algebra.

Proof. Proposition 2.1 of [37] implies that $B \cong p M_{n}(A) p$ for some $n$ and some projection $p \in M_{n}(A)$. So $B$ is a recursive subhomogeneous algebra by Lemma 1.12 and Corollary 1.11 .

Lemma 1.14. Let $A$ be a recursive subhomogeneous algebra with base spaces $X_{k}$, and let $Y$ be a compact Hausdorff space. Then $C(Y) \otimes A$ is a recursive subhomogeneous algebra, and has a decomposition with the same length as for $A$ and base spaces $Y \times X_{k}$. In particular, the topological dimension of this decomposition is at most $\operatorname{dim}(Y)$ times the topological dimension of $A$, and its maximum matrix size is the same as for $A$.

Proof. The first part is immediate from the definition. (It is also a special case of Theorem 3.4 of 32 .) For the last sentence one needs $\operatorname{dim}\left(Y \times X_{k}\right) \leq \operatorname{dim}(Y)+$ $\operatorname{dim}\left(X_{k}\right)$, which is Proposition 3.2.6 of [31].

\section{Characterization of Recursive subhomogeneous algebras}

In this section, we show that a separable unital $\mathrm{C}^{*}$-algebra has a recursive subhomogeneous decomposition with finite topological dimension if and only if there is a finite upper bound on the dimensions of irreducible representations and, for each $n$, the space of irreducible representations of dimension $n$ is finite dimensional. 
This result shows that there are many recursive subhomogeneous algebras. We further prove a related (but not as nice) characterization of recursive subhomogeneous algebras in the absence of finite dimensionality.

In this section, we use the modified covering dimension of a topological space $X$. This is the same as covering dimension, except that one only considers covers consisting of complements of the zero sets of continuous real-valued functions on $X$. See Definition 10.1.3 of [31, but note that this function is denoted $\partial \operatorname{im}(X)$ there, and we will simply write $\operatorname{dim}(X)$. For compact Hausdorff spaces, and more generally for normal spaces, this dimension agrees with the usual covering dimension (Proposition 10.1.6 of [31]), and for completely regular spaces this definition gives $\operatorname{dim}(X)=\operatorname{dim}(\beta X)$ (Theorem 10.1.4 of [31]). The difference will be relevant only in some of the results which are stated for not necessarily $\sigma$-compact spaces.

Lemma 2.1. Let $A$ be a recursive subhomogeneous algebra with total space $X$. Then the map $x \mapsto \operatorname{Ker}\left(\mathrm{ev}_{x}\right)$ defines a continuous bijection

$$
\coprod_{k=0}^{l}\left(X_{k} \backslash X_{k}^{(0)}\right) \rightarrow \operatorname{Prim}(A)
$$

whose restriction to each $X_{k} \backslash X_{k}^{(0)}$ is a homeomorphism onto its image. (We take $X_{0}^{(0)}=\varnothing$.) In particular, every irreducible representation of $A$ is unitarily equivalent to a representation of the form $\mathrm{ev}_{x}$ for some $x \in X$.

Proof. The proof is by induction on the length $l$. If the length is zero, then $A=$ $C\left(X, M_{n}\right)$ for some $X$ and $n$, and the result is obvious. So assume that the result is known for length $l$. Let $A=B \oplus_{C\left(X^{(0)}, M_{n}\right)} C\left(X, M_{n}\right)$, where $B$ is a recursive subhomogeneous algebra of length $l$. Consider the exact sequence

$$
0 \longrightarrow C_{0}\left(X \backslash X^{(0)}, M_{n}\right) \longrightarrow A \longrightarrow B \longrightarrow 0
$$

in which the first map is $f \mapsto(0, f)$ and the second is $(a, f) \mapsto a$. It shows that $\operatorname{Prim}(A)$ is the (nontopological) disjoint union of the closed set $\operatorname{Prim}(B)$ and the open set $\operatorname{Prim}\left(C_{0}\left(X \backslash X^{(0)}, M_{n}\right)\right) \cong X \backslash X^{(0)}$, and these two identifications are homeomorphisms onto their images. So the result for $B$ implies the result for $A$.

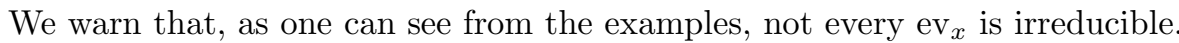

The next definition is a generalization of Definition 3.5.7 of [16] (which is only stated for vector bundles), and is presumably known in the generality in which we give it. I am grateful to Larry Brown for the suggestion that the finite type condition might be relevant here.

Definition 2.2. Let $X$ be a topological space and let $E$ be a locally trivial fiber bundle over $X$. Then $E$ is said to have finite type if there is a finite open cover $U_{1}, U_{2}, \ldots, U_{n}$ of $X$ such that $\left.E\right|_{U_{k}}$ is trivial for $1 \leq k \leq n$.

We prove that vector bundles over sufficiently nice finite-dimensional spaces have finite type. This is proved in Lemma 2.19 of [27, but that reference is possibly not widely available and in any case uses a slightly different definition of covering dimension.

Lemma 2.3. Let $X$ be a compact Hausdorff space with $\operatorname{dim}(X) \leq d<\infty$. Let $E$ be a vector bundle over $X$ with rank $r$. Then there exist $d+1$ open sets $V_{1}, V_{2}, \ldots, V_{d+1}$ $\subset X$ which cover $X$ and such that $\left.E\right|_{V_{k}}$ is trivial for $1 \leq k \leq d+1$. 
Proof. The method of proof of Theorem 2.5 of [15] shows that there is a finite complex $Y$ with $\operatorname{dim}(Y) \leq d$, a continuous function $f: X \rightarrow Y$, and a vector bundle $F$ over $Y$ with rank $r$, such that $f^{*}(F) \cong E$. (The argument reduces to the fact that $X$ is an inverse limit of compact metric spaces of dimension at most $d$, and such a space is in turn an inverse limit of finite complexes of dimension at most $d$.) Now Theorem 1.2.6 of [16] provides $d+1$ open sets $W_{1}, W_{2}, \ldots, W_{d+1} \subset Y$ which cover $Y$ and such that each connected component of each $W_{k}$ is contractible. Therefore, $\left.F\right|_{W_{k}}$ is trivial. The proof is completed by taking $V_{k}=f^{-1}\left(W_{k}\right)$ and noting that $\left.E\right|_{V_{k}} \cong f^{*}\left(\left.F\right|_{W_{k}}\right)$.

Lemma 2.4. Let $X$ be a locally compact $\sigma$-compact Hausdorff space. Then there exist open sets $U_{1}, U_{2}, \cdots \subset X$ which cover $X$, whose closures are compact, and such that $\bar{U}_{j} \cap \bar{U}_{k}=\varnothing$ for $|j-k|>1$.

Proof. Let $K_{1}, K_{2}, \cdots \subset X$ be a sequence of compact sets which covers $X$. We construct $U_{n}$ by induction on $n$, satisfying:

(1) $\bigcup_{k=1}^{n} U_{k} \supset K_{n}$.

(2) $\partial\left(\bigcup_{k=1}^{n-1} U_{k}\right) \subset U_{n}$.

(3) $\bar{U}_{j} \cap \bar{U}_{k}=\varnothing$ for $|j-k|>1$ and $1 \leq j, k \leq n$.

(4) $\bar{U}_{k}$ is compact for $1 \leq k \leq n$.

We start the induction by choosing $U_{1}$ to be any open set which contains $K_{1}$ and has compact closure.

Suppose given $U_{1}, U_{2}, \ldots, U_{n}$ as above. Choose an open set $V$ which contains $K_{n+1} \cup \bigcup_{k=1}^{n} \bar{U}_{k}$ and has compact closure. Define

$$
W=V \backslash \bigcup_{k=1}^{n-1} \bar{U}_{k} \quad \text { and } \quad L=\left(K_{n+1} \backslash \bigcup_{k=1}^{n} U_{k}\right) \cup \partial\left(\bigcup_{k=1}^{n} U_{k}\right) .
$$

Thus $W$ is open and $L$ is compact. Also $L \subset W$, because

$$
V \backslash \bigcup_{k=1}^{n} U_{k} \subset V \backslash \bigcup_{k=1}^{n-1} \bar{U}_{k} \text { and } \partial\left(\bigcup_{k=1}^{n} U_{k}\right) \subset \bigcup_{k=1}^{n} \bar{U}_{k} \backslash \bigcup_{k=1}^{n} U_{k} \subset W .
$$

Now choose an open set $U_{n+1}$ with compact closure such that $L \subset U_{n+1} \subset$ $\bar{U}_{n+1} \subset W$. Conditions (1), (2), and (4) are satisfied for $n+1$ by construction. For (3), we need only show that $\bar{U}_{n+1} \cap \bar{U}_{k}=\varnothing$ for $1 \leq k \leq n-1$. But this follows from the relation $W \cap \bigcup_{k=1}^{n-1} \bar{U}_{k}=\varnothing$. This completes the induction. The lemma is now proved because the sets $K_{n}$ cover $X$.

Lemma 2.5. Let $X$ be a locally compact $\sigma$-compact Hausdorff space with $\operatorname{dim}(X) \leq d<\infty$. Let $E$ be a vector bundle over $X$ with (constant) rank $r$. Then $E$ has finite type.

Proof. Choose open sets $U_{1}, U_{2}, \ldots \subset X$ satisfying the conclusion of Lemma 2.4, We have $\operatorname{dim}\left(\bar{U}_{k}\right) \leq d$ by Proposition 3.1.5 of [31]. (Since $X$ and $\bar{U}_{k}$ are normal, the modified covering dimension is the same as the usual one.) By Lemma 2.3 there exist open subsets $V_{k, 1}, V_{k, 2}, \ldots, V_{k, d+1} \subset \bar{U}_{k}$ which cover $\bar{U}_{k}$ and such that $\left.E\right|_{V_{k, l}}$ is trivial for $1 \leq l \leq d+1$. Now define

$$
W_{l}^{(0)}=\bigcup_{k \text { even }}\left(U_{k} \cap V_{k, l}\right) \quad \text { and } \quad W_{l}^{(1)}=\bigcup_{k \text { odd }}\left(U_{k} \cap V_{k, l}\right) .
$$


Each of these $2(d+1)$ sets is the union of disjoint open subsets of $X$ over which $E$ is trivial, since $U_{j} \cap U_{k}=\varnothing$ for $|j-k|>1$. Therefore, $E$ is trivial over each $W_{l}^{(i)}$. Since these sets cover $X$, the proof is complete.

The following equivalent conditions for finite type will be very useful.

Lemma 2.6. Let $X$ be a completely regular space, and let $E$ be a vector bundle over $X$ with (constant) rank $r$. Then the following are equivalent:

(1) E has finite type.

(2) There exists a vector bundle $H$ over $X$ such that $E \oplus H$ is trivial.

(3) There exists a vector bundle $F$ over $\beta X$, the Stone-Čech compactification of $X$, such that $\left.F\right|_{X} \cong E$.

(4) There exists a compactification $Y$ of $X$, and a vector bundle $F$ over $Y$, such that $\left.F\right|_{X} \cong E$.

Proof. The equivalence of (1) and (2) is the equivalence of conditions (1) and (3) of Proposition 3.5.8 of [16]. That (3) implies (4) is trivial. For (4) implies (2), observe that $F$ is a direct summand of a trivial bundle because $Y$ is compact. Restricting to $X$, we see that $E$ is a direct summand of a trivial bundle.

It remains to show that (2) implies (3). We may assume $E \oplus H=X \times \mathbf{C}^{n}$ for some $n$. Equip $X \times \mathbf{C}^{n}$ with the usual scalar product, and let $p$ be the orthogonal projection from $X \times \mathbf{C}^{n}$ to $E$. We may think of $p$ as a bounded continuous function from $X$ to $M_{n}$. Then there is a continuous function $q$ from $\beta X$ to $M_{n}$ which extends $p$. The values of $q$ must be projections, so $F=q\left(X \times \mathbf{C}^{n}\right)$ is a vector bundle over $\beta X$ such that $\left.F\right|_{X} \cong p\left(X \times \mathbf{C}^{n}\right) \cong E$.

Our next goal is a similar characterization of finite type for locally trivial $M_{n^{-}}$ bundles. We will in fact see that an $M_{n}$-bundle has finite type if and only if the underlying $n^{2}$-dimensional vector bundle does. We need several lemmas.

Lemma 2.7. Let $X$ be a compact Hausdorff space. Let $E$ be a vector bundle over $X$ with rank $r>0$. Then there exists a vector bundle $F$ over $X$ with rank $s>0$ such that $E \otimes F$ is trivial.

Proof. Use Theorem 10.1 in Chapter 10 of [11] to write $X=\lim _{\alpha} X_{\alpha}$ for finite

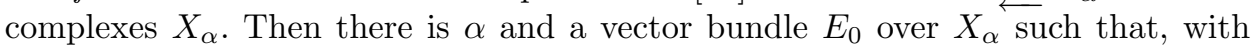
$f: X \rightarrow X_{\alpha}$ being the canonical map, we have $E \cong f^{*}\left(E_{0}\right)$. Write $X_{\alpha}$ as a disjoint union $X_{\alpha}=\coprod_{k=1}^{n} Y_{k}$ of connected finite complexes. Lemma 12 of [10] provides vector bundles $G_{k}$ over $Y_{k}$ with positive rank $s(k)$ such that $\left.G_{k} \otimes E\right|_{Y_{k}}$ is trivial. Set $t(k)=\prod_{l \neq k} s(l)$. Then $F_{0}=\coprod_{k=1}^{n} G_{k} \otimes\left(Y_{k} \times \mathbf{C}^{t(k)}\right)$ is a vector bundle over $X_{\alpha}$ with constant rank $s=\prod_{k=1}^{n} s(k)>0$ and such that $E_{0} \otimes F_{0}$ is trivial. Take $F=f^{*}\left(F_{0}\right)$.

Lemma 2.8. Let $X$ be a compact Hausdorff space, let $S$ be a dense subset of $X$, and let $n, N \in \mathbf{N}$. Let $x \mapsto P_{x}$ be a continuous function from $X$ to the space $L\left(M_{N}\right)$ of bounded operators on $M_{N}$. Suppose that, for all $x \in S$, the map $P_{x}$ is a conditional expectation from $M_{N}$ onto a subalgebra $A_{x} \cong M_{n}$ of $M_{N}$. Then $P_{x}$ is a conditional expectation from $M_{N}$ onto a subalgebra $A_{x} \cong M_{n}$ for every $x \in X$. Moreover, the assignment $x \mapsto A_{x}$ defines a locally trivial continuous field of $C^{*}$-algebras over $X$ with fiber $M_{n}$. 
Proof. It is easy to check that the set of $x \in X$ for which the range of $P_{x}$ is not a subalgebra is open in $X$, and hence empty. The other algebraic and norm properties of conditional expectations extend over $X$ in the same way. Similarly, the trace of $P_{x}$ is $n^{2}$ for all $x \in S$, hence for all $x \in X$. It follows that $P_{x}$ has rank exactly equal to $n^{2}$ for all $x \in X$.

Let

$$
V=\left\{x \in X: A_{x} ¥ M_{n}\right\} \subset X,
$$

and let $x \in V$. Since $\operatorname{dim}\left(A_{x}\right)=n^{2}$, it follows that there are $n+1$ nonzero mutually orthogonal projections $p_{1}, \ldots, p_{n+1} \in A_{x}$. Define $a_{k}(y)=P_{y}\left(p_{k}\right)$ for $y \in X$. Then the $a_{k}$ are continuous functions from $X$ to $M_{N}$, such that $a_{k}(y) \in A_{y}$ for all $y$. Standard functional calculus techniques (or Chapter 14 of [25]) show that, on some neighborhood of $x$, there are continuous mutually orthogonal projections $y \mapsto q_{1}(y), \ldots, y \mapsto q_{n+1}(y)$, whose values at $y$ are in the $\mathrm{C}^{*}$-subalgebra of $M_{N}$ generated by $a_{1}(y), \ldots, a_{n+1}(y)$. (This is semiprojectivity of $\mathbf{C}^{n+1}$.) Therefore, for $y$ close enough to $x$, there are $n+1$ nonzero mutually orthogonal projections $q_{1}(y), \ldots, q_{n+1}(y) \in A_{y}$. It follows that $A_{y} \nsucceq M_{n}$, so that $V$ is open. Since $V \cap S=\varnothing$, we have $A_{x} \cong M_{n}$ for all $x \in X$.

It remains to prove local triviality. Let $x \in X$. Choose a system $\left(e_{j, k}\right)_{1 \leq j, k \leq n}$ of matrix units for $A_{x}$. Using the conditional expectation $P_{x}$ and standard functional calculus techniques (or [25]) as in the previous paragraph, we may find a neighborhood $U$ of $x$ and continuous functions $f_{j, k}: V \rightarrow M_{N}$ for $1 \leq j, k \leq n$ such that $f_{j, k}(y) \in A_{y}, f_{j, k}(x)=e_{j, k}$, and $\left(f_{j, k}(y)\right)_{1 \leq j, k \leq n}$ is a system of matrix units. (This is semiprojectivity of $M_{n}$.) Reducing the size of $V$ if necessary, we may find a continuous function $s: V \rightarrow M_{N}$ such that

$$
s(y)^{*} s(y)=e_{1,1}, \quad s(y) s(y)^{*}=f_{1,1}(y), \quad \text { and } \quad s(x)=e_{1,1} .
$$

Then $y \mapsto w(y)=\sum_{j=1}^{n} f_{j, 1}(y) s(y) e_{1, j}$ is a continuous function from $V$ to the unitaries in $M_{N}$ such that $w e_{j, k} w^{*}=f_{j, k}(y)$. Thus $w A_{x} w^{*}=A_{y}$, and the bundle $y \mapsto A_{y}$ is trivial over $V$.

Proposition 2.9. Let $X$ be a completely regular space, and let $A$ be a locally trivial continuous field of $C^{*}$-algebras over $X$ with fiber $M_{n}$. Then the following are equivalent:

(1) A has finite type.

(2) A has finite type when regarded as a complex vector bundle over $X$ by forgetting structure.

(3) There exists a locally trivial continuous field $B$ of $C^{*}$-algebras over $\beta X$ such that $\left.B\right|_{X} \cong A$.

(4) There exists a compactification $Y$ of $X$, and a locally trivial continuous field $B$ of $C^{*}$-algebras $F$ over $Y$, such that $\left.B\right|_{X} \cong A$.

(5) There exists $k$ and a locally trivial continuous field $C$ of $C^{*}$-algebras over $X$ with fiber $M_{k}$ such that $A \otimes C$ is trivial.

Proof. We prove $(1) \Rightarrow(2) \Rightarrow(5) \Rightarrow(3) \Rightarrow(4) \Rightarrow(1)$. The implications (1) $\Rightarrow(2)$ and $(3) \Rightarrow(4)$ are immediate, and $(4) \Rightarrow(1)$ follows directly from compactness of $Y$ and local triviality of $B$.

We prove $(2) \Rightarrow(5)$. Define a scalar product in the fibers of $A$ by taking the Hilbert-Schmidt scalar product. Since $A$ has finite type as a vector bundle, $(1) \Rightarrow$ (4) of Lemma 2.6 provides a compactification $Y$ of $X$ and a vector bundle $F$ over 
$Y$ such that $\left.F\right|_{X} \cong A$. Lemma 2.7 then provides a vector bundle $H$ over $Y$ and an isomorphism $a: F \otimes H \rightarrow Y \times \mathbf{C}^{N}$ for some $N$. We may assume $H$ has a continuously varying scalar product and equip $Y \times \mathbf{C}^{N}$ with the usual scalar product. Then, replacing $a$ by $a\left(a^{*} a\right)^{-1 / 2}$, we may assume that $a$ is unitary.

Let $A^{\text {op }}$ denote the locally trivial continuous field over $X$ with fiber $M_{n}$ obtained by reversing the multiplication in every fiber. Then, as is well known, there is a natural *-isomorphism $A \otimes A^{\text {op }} \cong L(A)$, the locally trivial continuous field over $X$ with fiber $M_{n^{2}} \cong L\left(A_{x}\right)$ using the Hilbert space structure on $A_{x}$ from the previous paragraph. (Lacking a reference, we give a formula. Define $\pi_{x}: A_{x} \otimes A_{x}^{\text {op }} \rightarrow$ $L\left(A_{x}\right)$ by $\pi_{x}\left(a_{1} \otimes a_{2}\right)(c)=a_{1} c a_{2}$. One easily checks that this defines a unital *homomorphism. Simplicity of the domain $A_{x} \otimes A_{x}^{\text {op }}$ and a dimension count show that it is bijective.) With similar notation, we have

$$
L(A) \otimes L\left(\left.H\right|_{X}\right) \cong L\left(\left.A \otimes H\right|_{X}\right) \cong L\left(X \times \mathbf{C}^{N}\right) \cong X \times M_{N} .
$$

This is (5) with $C=A^{\text {op }} \otimes L\left(\left.H\right|_{X}\right)$.

It remains to prove $(5) \Rightarrow(3)$. By hypothesis, there exist $k$ and a locally trivial continuous field $C$ over $X$ with fiber $M_{k}$ such that $A \otimes C \cong M_{n k}$. For each $x \in X$, the tensor product decomposition $M_{n k} \cong A_{x} \otimes C_{x}$ gives a conditional expectation $P_{x}: M_{n k} \rightarrow A_{x}$. Then $x \mapsto P_{x}$ is a continuous function from $X$ to the operators of norm 1 on $M_{n k}$. Therefore, it extends to a continuous function, still called $x \mapsto P_{x}$, defined on $\beta X$. By Lemma 2.8 the assignment $x \mapsto P_{x}\left(M_{n k}\right)$ defines a locally trivial continuous field over $\beta X$ with fiber $M_{n}$, whose restriction to $X$ is obviously $A$.

Several more lemmas are needed before we can prove the characterization theorems.

Lemma 2.10. Let $X$ be a second countable locally compact Hausdorff space, and let $Y$ be a compactification of $X$. Let $A$ be a locally trivial continuous field of $C^{*}$ algebras over $Y$ with fiber $M_{n}$, and let $D$ be a separable $C^{*}$-subalgebra of the section algebra $\Gamma\left(\left.A\right|_{Y \backslash X}\right)$. Then there are a compactification $Z$ of $X$, a surjective map $h: Y \rightarrow Z$ which is the identity on $X$, and a locally trivial continuous field $B$ over $Z$ with fiber $M_{n}$, such that:

(1) $Z$ is second countable.

(2) $\operatorname{dim}(Z)=\operatorname{dim}(X)$.

(3) There is an isomorphism $\varphi:\left.\left.h^{*}(B)\right|_{Y \backslash X} \cong A\right|_{Y \backslash X}$ such that the range of the induced map $\sigma: \Gamma\left(\left.B\right|_{Z \backslash X}\right) \rightarrow \Gamma\left(\left.A\right|_{Y \backslash X}\right)$ contains D. $($ Here $\sigma(b)(x)=$ $\varphi_{x}(b(h(x)))$.)

Proof. We first find a compactification $Z_{0}$ with $h_{0}: Y \rightarrow Z_{0}$ such that all of the conclusion except part (2) holds. By (4) implies (5) of Proposition 2.9, applied to the compact space $Y$, there exists a locally trivial continuous field $C$ over $Y$ with fiber $M_{k}$ and an isomorphism $\mu_{0}: A \otimes C \rightarrow Y \times M_{n k}$. This map induces a homomorphism $\mu: \Gamma\left(\left.A\right|_{Y \backslash X}\right) \rightarrow C\left(Y \backslash X, M_{n k}\right)$. It also gives a continuous function $x \mapsto P_{x}$ from $Y$ to the set of conditional expectations from $M_{n k}$ onto subalgebras, such that $P_{x}\left(M_{n k}\right)=\left(\mu_{0}\right)_{x}\left(A_{x} \otimes 1\right)$ for all $x \in Y$. For $x, y \in Y \backslash X$ define $x \sim y$ to mean $P_{x}=P_{y}$ and $\mu(a)(x)=\mu(a)(y)$ for all $a \in D$. This defines an equivalence relation on $Y \backslash X$, and we extend it to all of $Y$ by taking $x \sim y$ exactly when $x=y$ for $x, y \in X$. Let $Z_{0}$ be the maximal ideal space of the commutative unital 
$\mathrm{C}^{*}$-algebra

$$
R=\{f \in C(Y): f(x)=f(y) \text { whenever } x \sim y\} .
$$

Let $h_{0}: Y \rightarrow Z_{0}$ be the continuous surjective map induced by the inclusion of $R$ in $C(Y)$. Note that $h_{0}$ sends $X$ homeomorphically onto $h_{0}(X) \subset Z_{0}$, and we can thus identify $X$ with $h_{0}(X)$. Moreover, $h_{0}(x)=h_{0}(y)$ if and only if $x \sim y$.

The map $x \mapsto P_{x}$ defines a continuous function $x \mapsto \bar{P}_{x}$ from $Z_{0}$ to the set of conditional expectations from $M_{n k}$ onto subalgebras. It follows from Lemma 2.8 that $x \mapsto \bar{P}_{x}\left(M_{n k}\right)$ defines a locally trivial continuous field $B_{0}$ over $Z_{0}$ with fiber $M_{n}$. Since $x \mapsto P_{x}\left(M_{n k}\right)$ is just $A$ (the isomorphism being obtained from $\mu_{0}$ ), there is an obvious isomorphism $h_{0}^{*}\left(B_{0}\right) \cong A$. The range of the induced map from $\Gamma\left(\left.B_{0}\right|_{Z_{0} \backslash X}\right)$ to $\Gamma\left(\left.A\right|_{Y \backslash X}\right)$ consists exactly of all sections $a$ of $\left.A\right|_{Y \backslash X}$ such that $\left(\mu_{0}\right)_{x}(a(x))=\left(\mu_{0}\right)_{y}(a(y))$ whenever $x \sim y$. In particular, it contains $D$, as required.

We show $Z_{0}$ is second countable. Extend $x \mapsto P_{x}$ to a continuous function on $Y$ (whose values need no longer be conditional expectations). Let

$$
\widetilde{D}=\left\{a \in C\left(Y, M_{n k}\right):\left.a\right|_{Y \backslash X} \in \mu(D)\right\} .
$$

Then $\widetilde{D}$ is separable because $X$ is second countable and there is an exact sequence

$$
0 \longrightarrow C_{0}\left(X, M_{n k}\right) \longrightarrow \widetilde{D} \longrightarrow D \longrightarrow 0 .
$$

The extended function $x \mapsto P_{x}$ and the elements of $\widetilde{D}$ are all images of functions on $Z_{0}$, and together they separate the points of $Z_{0}$. This implies that $Z_{0}$ is second countable.

We now modify $Z_{0}$ so as to obtain condition (2) as well. By Proposition 10.3.11 of [31, there exist a compact Hausdorff space $Z$ and continuous surjections $h: Y \rightarrow$ $Z$ and $g: Z \rightarrow Z_{0}$ such that $h_{0}=g \circ h, \operatorname{dim}(Z)=\operatorname{dim}(Y)$, and $Z$ is also second countable. The proof is completed by taking $B=g^{*}\left(B_{0}\right)$.

Lemma 2.11. Let $X$ be a locally compact Hausdorff space, and let $A$ be a locally trivial continuous field over $X$ with fiber $M_{n}$ which has finite type. Let $\Gamma_{0}(A)$ be the $C^{*}$-algebra of continuous sections of $A$ which vanish at infinity on $X$. Let

$$
0 \longrightarrow \Gamma_{0}(A) \longrightarrow C \longrightarrow D \longrightarrow 0
$$

be an exact sequence with $C$ and $D$ unital. Then there is a compactification $Y$ of $X$, a locally trivial continuous field $B$ over $Y$ with fiber $M_{n}$, and a unital homomorphism $\varphi: D \rightarrow \Gamma\left(\left.B\right|_{Y \backslash X}\right)$ such that:

(1) $\operatorname{dim}(Y)=\operatorname{dim}(X)$.

(2) $\left.B\right|_{X} \cong A$.

(3) There is an isomorphism $\psi: D \oplus_{\Gamma\left(\left.B\right|_{Y \backslash X}\right)} \Gamma(B) \rightarrow C$ which fits into a commutative diagram with exact rows

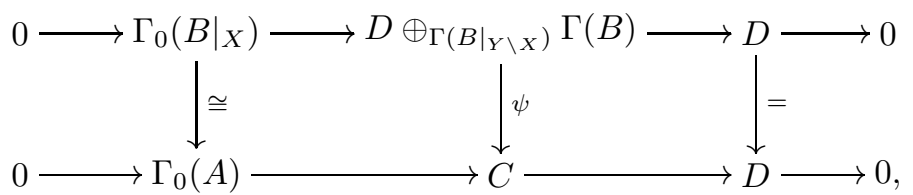

in which the first vertical map is induced by the isomorphism of (2) and the pullback is via $\varphi$ and the restriction of sections to $Y \backslash X \subset X$.

Moreover, if $X$ is second countable and $D$ is separable, then $Y$ may be chosen to be second countable. 
Proof. We begin by identifying the multiplier algebra of $\Gamma_{0}(A)$. By parts (3) and (5) of Proposition 2.9, there is a locally trivial continuous field $B_{0}$ over $\beta X$ with fiber $M_{n}$ such that $\left.B_{0}\right|_{X} \cong A$, and, moreover, $B_{0}$ is a subbundle of a trivial bundle $\beta X \times M_{N}$ for some $N$. All bounded continuous sections of $A$ extend uniquely to sections of $\beta X \times M_{N}$, and clearly the sections one gets in this way are exactly the continuous sections of $B_{0}$. It now follows from Theorem 3.3 of [1] that $M\left(\Gamma_{0}(A)\right)$ can be identified with $\Gamma\left(B_{0}\right)$.

The exact sequence of the hypothesis thus yields homomorphisms

$$
\tau: C \rightarrow M\left(\Gamma_{0}(A)\right)=\Gamma\left(B_{0}\right) \quad \text { and } \quad \bar{\tau}: D \rightarrow \Gamma\left(B_{0}\right) / \Gamma_{0}(A)=\Gamma\left(\left.B_{0}\right|_{\beta X \backslash X}\right) .
$$

In the general case, we take $Y=\beta X, B=B_{0}$, and $\varphi=\bar{\tau}$. We have $\operatorname{dim}(\beta X)=$ $\operatorname{dim}(X)$ by Theorem 10.1.4 of 31] (recall our definition of $\operatorname{dim})$. We now construct $\psi$. Let $\pi: C \rightarrow D$ be the quotient map, and let $\rho: \Gamma\left(B_{0}\right) \rightarrow \Gamma\left(\left.B_{0}\right|_{\beta X \backslash X}\right)$ be restriction. By definition, if $c \in C$, then $\rho \circ \tau(c)=\varphi \circ \pi(c)$, so $\psi=(\varphi, \rho)$ is a well defined homomorphism making the diagram in (3) commute. Moreover, $\psi$ is an isomorphism by the Five Lemma.

In the second countable case, we apply Lemma 2.10 to $B_{0}, \beta X$, and $\bar{\tau}(D) \subset$ $\Gamma\left(\left.B_{0}\right|_{\beta X \backslash X}\right)$, obtaining a second countable compactification $Y$ of $X$ with $\operatorname{dim}(Y)=$ $\operatorname{dim}(\beta X)=\operatorname{dim}(X)$, and a locally trivial continuous field $B$ over $Y$, such that $\Gamma\left(\left.B\right|_{Y \backslash X}\right)$ can be canonically identified with a subalgebra of $\Gamma\left(\left.B_{0}\right|_{\beta X \backslash X}\right)$ which contains $\bar{\tau}(D)$. These changes from the case above do not change the pullback $D \oplus_{\Gamma\left(\left.B\right|_{Y \backslash X}\right)} \Gamma(B)$, so the lemma is proved in this case also.

We let $\operatorname{Prim}(A)$ denote the primitive ideal space of a $\mathrm{C}^{*}$-algebra $A$, and we let $\operatorname{Prim}_{n}(A)$ denote the subspace of $\operatorname{Prim}(A)$ consisting of the kernels of $n$-dimensional representations of $A$. We summarize some of the standard facts. We refer to $E$ as in (4) in the following theorem as the induced continuous field over $\operatorname{Prim}_{n}(A)$.

Theorem 2.12. For each finite $n$ :

(1) $\bigcup_{k \leq n} \operatorname{Prim}_{k}(A)$ is closed in $\operatorname{Prim}(A)$.

(2) $\operatorname{Prim}_{n}(A)$ is open in $\bigcup_{k \leq n} \operatorname{Prim}_{k}(A)$.

(3) $\operatorname{Prim}_{n}(A)$ is locally compact Hausdorff.

(4) There is a locally trivial continuous field $E$ over $\operatorname{Prim}_{n}(A)$ with fiber $M_{n}$ such that the subquotient of $A$ corresponding to $\operatorname{Prim}_{n}(A)$ is isomorphic to $\Gamma_{0}(E)$.

Proof. Parts (1) and (2) are Proposition 3.6.3(i) of [9], and part (3) is Proposition 3.6.4(i) of [9]. Part (4) is Theorem 3.2 on page 249 of [14]. (Also see Theorems 3 and 5 of 43 .)

Proposition 2.13. Let $A$ be a unital $C^{*}$-algebra. Suppose that there is $N \in \mathbf{N}$ such that all irreducible representations of $A$ have dimension at most $N$, and suppose that the induced continuous fields over the subspaces $\operatorname{Prim}_{n}(A)$ (Theorem 2.12(4)) all have finite type. Then $A$ has a recursive subhomogeneous decomposition which, when given in the standard notation (see Definition 1.2) has the following properties:

(1) $n(0) \leq n(1) \leq \cdots \leq n(l)$.

(2) $\operatorname{dim}\left(X_{k}\right) \leq \operatorname{dim}\left(\operatorname{Prim}_{n(k)}(A)\right)$ for $0 \leq k \leq l$.

(3) $X_{k} \backslash X_{k}^{(0)}$ is dense in $X_{k}$ for $1 \leq k \leq l$. 
In particular, it has topological dimension at most $\max _{1 \leq n \leq N} \operatorname{dim}\left(\operatorname{Prim}_{n}(A)\right)$ and maximum matrix size at most $N$.

If $A$ is separable, then the recursive subhomogeneous decomposition can be chosen so that, in addition:

(4) Every $C^{*}$-algebra in the recursive subhomogeneous decomposition is separable.

Proof. Using Proposition 1.7 and Theorem 2.12, this follows from Lemma 2.11 by induction. (The application of part (4) of the conclusion of Proposition 1.7 is justified as follows: In the application of Lemma 2.11, we use a compactification of $\operatorname{Prim}_{n}(A)$, and $\operatorname{Prim}_{n}(A)$ is dense in a compactification by definition.)

Lemma 2.14. Let $X$ be a metric space, and let $E$ be a vector bundle over $X$ with rank $r$. Suppose that there exist open sets $U_{1}, \ldots, U_{n} \subset X$ and closed sets $L_{1}, \ldots, L_{n} \subset X$, such that the sets $U_{k} \cap L_{k}$ cover $X$ and $\left.E\right|_{U_{k} \cap L_{k}}$ has finite type. Then $E$ has finite type.

Proof. Applying the definition of finite type, we may immediately reduce to the case in which $\left.E\right|_{U_{k} \cap L_{k}} \cong\left(U_{k} \cap L_{k}\right) \times \mathbf{C}^{r}$ (but possibly with $n$ larger). Since $X$ is metric, $U_{k}$ is paracompact. Moreover, $U_{k} \cap L_{k}$ is closed in $U_{k}$. By paracompactness, $\left.E\right|_{U_{k}}$ has a scalar product, and by the usual methods we may assume that there is a unitary isomorphism $u:\left.E\right|_{U_{k} \cap L_{k}} \rightarrow\left(U_{k} \cap L_{k}\right) \times \mathbf{C}^{r}$. For each $x \in U_{k}$ choose an open set $V_{x} \subset U_{k}$ with $x \in V_{x}$ and a unitary trivialization $v_{x}:\left.E\right|_{V_{x}} \rightarrow V_{x} \times \mathbf{C}^{r}$. Then $v_{x} u^{*}$ defines a continuous function from $V_{x} \cap L_{k}$ to the unitaries on $\mathbf{C}^{r}$, and so the Tietze Extension Theorem gives a continuous function $a_{x}: V_{x} \rightarrow M_{r}$ which extends this function. Choose a locally finite partition of unity $\left(f_{\alpha}\right)_{\alpha \in I}$ subordinate to the open cover $\left(V_{x}\right)_{x \in U_{k}}$ of $U_{k}$, with $\operatorname{supp}\left(f_{\alpha}\right) \subset V_{x(\alpha)}$. Define a morphism of vector bundles $a:\left.E\right|_{U_{k}} \rightarrow U_{k} \times \mathbf{C}^{r}$ by $a=\sum_{\alpha \in I} f_{\alpha} a_{x(\alpha)}^{*} v_{x(\alpha)}$. Then $\left.a\right|_{U_{k} \cap L_{k}}=u$. Let $W_{k}=\left\{x \in U_{k}: a(x)\right.$ is invertible $\}$, which is an open subset of $X$ containing $U_{k} \cap L_{k}$ and over which $E$ is trivial. Since the sets $W_{k}$ cover $X$, this shows that $E$ has finite type.

Theorem 2.15. Let $A$ be a separable unital $C^{*}$-algebra, and let $N \in \mathbf{N}$. The following are equivalent:

(1) A has a recursive subhomogeneous decomposition with maximum matrix size at most $N$.

(2) A has a recursive subhomogeneous decomposition with maximum matrix size at most $N$ and whose total space is second countable.

(3) All irreducible representations of $A$ have dimension at most $N$, and for $1 \leq n \leq N$ the induced continuous field on $\operatorname{Prim}_{n}(A)$ has finite type.

Theorem 2.16. Let $A$ be a separable unital $C^{*}$-algebra, and let $N, d \in \mathbf{N}$. The following are equivalent:

(1) A has a recursive subhomogeneous decomposition with maximum matrix size at most $N$ and topological dimension at most $d$.

(2) A has a recursive subhomogeneous decomposition with maximum matrix size at most $N$ and topological dimension at most $d$, whose total space is second countable.

(3) All irreducible representations of $A$ have dimension at most $N$, and for $1 \leq n \leq N$ we have $\operatorname{dim}\left(\operatorname{Prim}_{n}(A)\right) \leq d$. 
It is not entirely clear (beyond Proposition 2.13) what happens in the absence of separability.

Proof of Theorems 2.15 and 2.16. In both cases, $(2) \Rightarrow(1)$ is trivial. In Theorem 2.15. (3) $\Rightarrow(2)$ follows from Proposition 2.13. In Theorem 2.16, one uses in addition Lemma 2.5 and Proposition 2.9.

We prove $(1) \Rightarrow(3)$. The condition on dimensions of representations is immediate from Lemma 2.1. For the other parts, let a recursive subhomogeneous decomposition for $A$ be given as in the notation of Definition 1.2. Apply Lemma 2.1 and intersect everything with $\operatorname{Prim}_{n}(A)$. The result is a continuous bijection

$$
\coprod_{n(k)=n}\left(X_{k} \backslash X_{k}^{(0)}\right) \rightarrow \operatorname{Prim}_{n}(A)
$$

whose restriction to each $X_{k} \backslash X_{k}^{(0)}$ is a homeomorphism onto its image $Y_{k}$. Moreover, $Y_{k} \subset \operatorname{Prim}_{n}(A)$ is locally compact, and hence is the intersection of a closed set, say $L_{k}$, and an open set, say $U_{k}$, in $\operatorname{Prim}_{n}(A)$. The subquotient of $A$ corresponding to $Y_{k}$ is just $C_{0}\left(X_{k} \backslash X_{k}^{(0)}, M_{n}\right)$. With $E$ being the induced continuous field on $\operatorname{Prim}_{n}(A)$, this shows that $\left.E\right|_{Y_{k}}$ is trivial. Now $\operatorname{Prim}_{n}(A)$ is second countable and locally compact (in particular, regular), and so the version of the Urysohn Metrization Theorem given in Theorem 4.4.1 of [28] implies it is metrizable. Therefore, Lemma 2.14implies that $E$ has finite type. This proves $(1) \Rightarrow(3)$ in Theorem 2.15.

For the corresponding part of Theorem 2.16, note that $X_{k} \backslash X_{k}^{(0)} \cong Y_{k}$ is an $F_{\sigma^{-}}$ set in $X_{k}$. Therefore, $\operatorname{dim}\left(Y_{k}\right) \leq \operatorname{dim}\left(X_{k}\right) \leq d$ by the remark after Proposition 3.5.4 of [31. Since $\operatorname{Prim}_{n}(A)$ is the union of finitely many such sets, and each is also an $F_{\sigma}$-set in $\operatorname{Prim}_{n}(A)$, Proposition 3.5.3 of [31] implies $\operatorname{dim}\left(\operatorname{Prim}_{n}(A)\right) \leq d$.

\section{Closure properties}

In the first section, we saw that the class of recursive subhomogeneous algebras is closed under finite direct sums, tensor products with $M_{n}$ and commutative $\mathrm{C}^{*}$-algebras, and passage to corners. In this section we extend that list in the separable case, using the characterization theorems of the previous section. We show that quotients, pullbacks, and tensor products of separable recursive subhomogeneous algebras are again separable recursive subhomogeneous algebras. Moreover, a quotient of a separable recursive subhomogeneous algebra with finite topological dimension is again an algebra of the same kind. We also give an example of a separable unital $\mathrm{C}^{*}$-algebra which has a finite upper bound on the dimensions of its irreducible representations but is not a recursive subhomogeneous algebra. Like all separable algebras with such an upper bound, this example is even a unital subalgebra of a separable homogeneous $\mathrm{C}^{*}$-algebra.

The first several results are corollaries of Theorems 2.15 and 2.16 .

Proposition 3.1. Let $A$ be a separable recursive subhomogeneous algebra, and let $I$ be an ideal in $A$. Then $A / I$ is a recursive subhomogeneous algebra. Moreover, $A / I$ has a decomposition in which the topological dimension and maximum matrix size are no larger than for $A$.

Proof. Condition (3) in Theorems 2.15 and 2.16 passes to quotients. (We have $\operatorname{dim}\left(\operatorname{Prim}_{n}(A / I)\right) \leq \operatorname{dim}\left(\operatorname{Prim}_{n}(A)\right)$ by Proposition 3.1.5 of 31], since $\operatorname{Prim}_{n}(A / I)$ is a closed subset of $\operatorname{Prim}_{n}(A)$.) 
Proposition 3.2 (Compare with Theorem 11.4 of [32]). Let $A$ and $B$ be separable recursive subhomogeneous algebras, and let $\varphi: A \rightarrow C$ and $\rho: B \rightarrow C$ be homomorphisms with $\varphi$ unital and $\rho$ surjective. Then $A \oplus_{C} B$ is a recursive subhomogeneous algebra. Moreover, $A \oplus_{C} B$ has a decomposition in which the topological dimension and maximum matrix size are each no larger than the maximum of the corresponding quantities for $A$ and $B$.

Proof. We have an exact sequence

$$
0 \longrightarrow \operatorname{Ker}(\rho) \longrightarrow A \oplus_{C} B \longrightarrow B \longrightarrow 0
$$

(as in the proof of Lemma 2.1). So $\operatorname{Prim}\left(A \oplus_{C} B\right)$ is the (nontopological) disjoint union of the open set $\operatorname{Prim}(\operatorname{Ker}(\rho))$ (also an open subset of $\operatorname{Prim}(A))$ and the closed set $\operatorname{Prim}(B)$. It is clear that the dimensions of irreducible representations of $A \oplus_{C} B$ can be no larger than for $A$ and $B$. There is a similar (nontopological) partition of $\operatorname{Prim}_{n}\left(A \oplus_{C} B\right)$ as the disjoint union of homeomorphic copies of $\operatorname{Prim}_{n}(\operatorname{Ker}(\rho)) \subset$ $\operatorname{Prim}_{n}(A)$ and $\operatorname{Prim}_{n}(B)$. Everything is metrizable. The induced continuous field $E$ on $\operatorname{Prim}_{n}\left(A \oplus_{C} B\right)$ restricts to the induced continuous fields on $\operatorname{Prim}_{n}(\operatorname{Ker}(\rho))$ and $\operatorname{Prim}_{n}(B)$, which have finite type, so $E$ has finite type by Lemma 2.14. Also,

$$
\operatorname{dim}\left(\operatorname{Prim}_{n}\left(A \oplus_{C} B\right)\right) \leq \max \left(\operatorname{dim}\left(\operatorname{Prim}_{n}(A)\right), \operatorname{dim}\left(\operatorname{Prim}_{n}(B)\right)\right)
$$

by the same reasoning as in the last paragraph of the proofs of Theorems 2.15 and 2.16 .

We will see in Example 3.6 below that subalgebras of separable recursive subhomogeneous algebras need not be recursive subhomogeneous algebras. However, unitized ideals are.

Corollary 3.3. Let $A$ be a separable recursive subhomogeneous algebra, and let $I \subset A$ be an ideal. Then the unitization $I^{+}$is a recursive subhomogeneous algebra, with topological dimension and maximum matrix size no larger than for $A$.

Proof. We can write $I^{+}=\mathbf{C} \oplus_{A / I} A$ using obvious maps, and the result then follows from Proposition 3.2 .

Proposition 3.4 (Compare with Theorem 11.8 of [32]). Let $A$ and $B$ be separable recursive subhomogeneous algebras. Then $A \otimes B$ is a recursive subhomogeneous algebra, with topological dimension at most the sum of the topological dimensions of $A$ and of $B$, and with maximum matrix size at most the product of the maximum matrix sizes of $A$ and of $B$.

Proof. The proof is by induction on the length of a decomposition of $B$. If $B=$ $C\left(X, M_{n}\right)$, then the result is immediate from Lemmas 1.12 and 1.14

Suppose therefore that the conclusion holds for some $B$, and consider

$$
A \otimes\left(B \oplus_{C\left(X^{(0)}, M_{n}\right)} C\left(X, M_{n}\right)\right) \cong(A \otimes B) \oplus_{A \otimes C\left(X^{(0)}, M_{n}\right)}\left(A \otimes C\left(X, M_{n}\right)\right) .
$$

Then $A \otimes B$ and $A \otimes C\left(X, M_{n}\right)$ are recursive subhomogeneous algebras satisfying the required bounds on the topological dimension and maximum matrix size, by the induction hypothesis and by the initial case of the induction respectively. Proposition 3.2 therefore implies that $A \otimes\left(B \oplus_{C\left(X^{(0)}, M_{n}\right)} C\left(X, M_{n}\right)\right)$ is a recursive subhomogeneous algebra satisfying the same bounds. 
We now give an example of a separable unital $\mathrm{C}^{*}$-algebra whose irreducible representations all have dimension at most 2 , but which is not a recursive subhomogeneous algebra.

Example 3.5. From Example 4.6 of 33 and the discussion following it, we obtain a complex line bundle $L$, not of finite type, over the second countable locally compact space $X=\coprod_{n=1}^{\infty} \mathbf{C} P^{n}$. Let $E=L \oplus(X \times \mathbf{C})$, and let $A$ be the unitization $\Gamma_{0}(L(E))^{+}$. Clearly, $A$ has a single one-dimensional representation, and its other irreducible representations all have dimension 2. Moreover, $\operatorname{Prim}_{2}(A)=X$ and the induced continuous field is $L(E)$. As a vector bundle, $L(E) \cong E \otimes E^{*}$, so that it contains $L \otimes(X \times \mathbf{C})^{*} \cong L$ as a subbundle. Since $L$ does not have finite type, neither does $L(E)$. (Use Lemma 2.6(2).) So $L(E)$ does not have finite type as an $M_{2}$-bundle, by Proposition 2.9. Therefore, $A$ does not have a recursive subhomogeneous decomposition by Theorem 2.15.

It is perhaps interesting to point out that this example is a direct limit of recursive subhomogeneous algebras, namely $\Gamma_{0}(L(E))^{+} \cong \lim \Gamma\left(L\left(\left.E\right|_{X_{n}}\right)\right)^{+}$, with $X_{n}=\coprod_{k=1}^{n} \mathbf{C} P^{k} \subset X$.

If $A$ is a separable unital $\mathrm{C}^{*}$-algebra which has a finite upper bound on the dimensions of its irreducible representations, then there is a unital embedding of $A$ as a subalgebra of a $\mathrm{C}^{*}$-algebra $C\left(Y, M_{n}(C)\right)$ for some finite $n$ and some compact metric space $Y$. See IV.1.4.3 of [4]. (I am grateful to Bruce Blackadar for pointing this out.) Example 3.5 thus shows that unital subalgebras of separable recursive subhomogeneous algebras, even of separable homogeneous $\mathrm{C}^{*}$-algebras, need not be recursive subhomogeneous algebras. The space $Y$ in this result need not be very nice, so we give a here a direct construction in which $Y$ can be taken to be the Cantor set. (In fact, combining the result quoted above with the method shown here, one can easily show that $Y$ in this result can always be taken to be the Cantor set.)

Example 3.6. Let $Y$ be the Cantor set. Then the algebra in Example 3.5 has a unital embedding into $C\left(Y, M_{2}\right)$.

To prove this, choose a continuous surjective function $f$ from $Y$ to the one point compactification $X^{+}$of $X$. (See, for example, Problem O part (e) in Chapter 5 of [19].) Set $Y_{n}=f^{-1}\left(\mathbf{C} P^{n}\right)$, so that the sets $Y_{n}$ are disjoint compact subsets of $Y$, and let $f_{n}=\left.f\right|_{Y_{n}}$. Then $\Gamma\left(L\left(\left.E\right|_{\mathbf{C} P^{n}}\right)\right)$ can be canonically identified with the set of sections $a$ of $L\left(f_{n}^{*}\left(\left.E\right|_{\mathbf{C} P^{n}}\right)\right)$ satisfying $a\left(y_{1}\right)=a\left(y_{2}\right)$ whenever $f_{n}\left(y_{1}\right)=f_{n}\left(y_{2}\right)$. (Recall that $f_{n}^{*}\left(\left.E\right|_{\mathbf{C} P^{n}}\right)_{y_{1}}=f_{n}^{*}\left(\left.E\right|_{\mathbf{C} P^{n}}\right)_{y_{2}}$ when $f_{n}\left(y_{1}\right)=f_{n}\left(y_{2}\right)$.) This identification extends in an obvious way to $\Gamma_{0}(L(E))$. However, $F=\left(\left.f\right|_{f^{-1}(X)}\right)^{*}(E)$ is trivial, because it is a bundle over the totally disconnected space $f^{-1}(X)$. Therefore, we obtain unital inclusions $\Gamma_{0}(L(E))^{+} \subset \Gamma_{0}(L(F))^{+} \subset C\left(Y, M_{2}\right)$.

\section{Cancellation in Recursive subhomogeneous algebras}

There are three basic theorems related to cancellation and subbundles of vector bundles on finite-dimensional compact spaces. Roughly, they are that a vector bundle of sufficiently large rank contains a trivial summand, that two stably isomorphic vector bundles of sufficiently large rank are actually isomorphic, and that if the difference between the ranks of two vector bundles is sufficiently large, then the smaller one is a direct summand in the bigger one. In each case, "sufficiently large" means at least about half the dimension of the space. See Section 9.1 of [16], 
and see Theorem 2.5 of [15], Lemma 3.4 of [26], and Lemma 1.5 of [34] for restatements in terms of projections in the $\mathrm{C}^{*}$-algebras $C\left(X, M_{n}\right)$. (Note that [16] contains a slightly weaker version of the third result, and that in the context of [16] the third result as stated above essentially contains the first.) In this section, we generalize the second and third results to recursive subhomogeneous algebras of finite topological dimension, and also prove an analogous result for unitaries. By applying the characterization results of Section 2, we then obtain results for type 1 $\mathrm{C}^{*}$-algebras whose irreducible representations have bounded dimension. In 35] and [36, these results will be applied to cancellation and related problems in direct limits of recursive subhomogeneous algebras.

The first result fails, because there might not be any trivial bundles of small rank. Indeed, the recursive subhomogeneous algebras in the direct system implicit in Example 4.8 of [35] have arbitrarily large minimum matrix size but no nontrivial projections. To prove the other two results, we first prove relative versions for vector bundles over finite complexes. Next, we switch to the $\mathrm{C}^{*}$-algebra context (that is, $C\left(X, M_{n}\right)$ ) and simultaneously generalize to arbitrary compact spaces. The proofs for recursive subhomogeneous algebras can then be done by induction. The results for unitaries can be gotten from the same preliminary results as those for projections.

In this section, we use the notation $p \sim q$ for Murray-von Neumann equivalence, and $p \precsim q$ to mean that $p$ is Murray-von Neumann equivalent to a subprojection of $q$. We let $U(A)$ denote the unitary group of a unital $\mathrm{C}^{*}$-algebra $A$, and we let $U_{0}(A)$ be the identity component of $U(A)$. We write (as usual) $\mathrm{GL}_{n}(\mathbf{C})$ for the invertible group $\operatorname{inv}\left(M_{n}\right)$.

Parts (1) and (2) of Proposition 4.1 are relative versions of Theorems 9.1.2 and 9.1.5 of [16], except that part (2) is generalized in the manner of Theorem 2.5 of [15], Lemma 3.4 of [26], and Lemma 1.5 of [34]. In part (2), the homotopy condition in the hypotheses is necessary. (For $d$ even, take $X$ to be the closed unit ball in $\mathbf{R}^{d}$, take $Y$ to be its boundary $S^{d-1}$, take $F=0$, take $E_{1}=E_{2}$ to be trivial and suffiently large, and take $a_{0}$ to represent a nontrivial element of $K^{1}\left(S^{d-1}\right)$.) The homotopy condition in the conclusion is then necessary for the proposition to be useful in induction arguments.

Proposition 4.1. Let $Y$ be a connected compact Hausdorff space, and let $X$ be a connected compact Hausdorff space obtained from $Y$ by attaching finitely many cells of dimension at most $d$. (In particular, the pair $(X, Y)$ could be a relative $C W$ complex of dimension at most d.)

(1) Let $E$ and $F$ be (complex) vector bundles over $X$ with ranks satisfying $\operatorname{rank}(E)-\operatorname{rank}(F) \geq \frac{1}{2}(d-1)$. Let $a_{0}:\left.\left.F\right|_{Y} \rightarrow E\right|_{Y}$ be an isomorphism of $\left.F\right|_{Y}$ with a subbundle of $\left.E\right|_{Y}$. Then there exists an isomorphism a of $F$ with a subbundle of $E$ such that $\left.a\right|_{Y}=a_{0}$.

(2) Let $E_{1}, E_{2}$, and $F$ be vector bundles over $X$ with $\operatorname{rank}\left(E_{1}\right) \geq \frac{1}{2} d$. Let $b: E_{1} \oplus$ $F \rightarrow E_{2} \oplus F$ be an isomorphism, let $a_{0}:\left.\left.E_{1}\right|_{Y} \rightarrow E_{2}\right|_{Y}$ be an isomorphism, and let $t \mapsto c_{t}^{(0)}$ be a homotopy of isomorphisms $c_{t}^{(0)}:\left.\left.\left(E_{1} \oplus F\right)\right|_{Y} \rightarrow\left(E_{2} \oplus F\right)\right|_{Y}$ such that $c_{0}^{(0)}=\left.a_{0} \oplus \operatorname{id}_{F}\right|_{Y}$ and $c_{1}^{(0)}=\left.b\right|_{Y}$. Then there exists an isomorphism $a: E_{1} \rightarrow E_{2}$ such that $\left.a\right|_{Y}=a_{0}$, and a homotopy of isomorphisms $c_{t}: E_{1} \oplus F \rightarrow E_{2} \oplus F$ such that

$$
c_{0}=a \oplus \operatorname{id}_{F}, \quad c_{1}=b, \quad \text { and }\left.\quad c_{t}\right|_{Y}=c_{t}^{(0)} .
$$


Proof. By an obvious induction, we may assume $X$ is obtained from $Y$ by attaching a single cell of dimension $n \leq d$, that is, $X=Y \cup_{f} D^{n}$, where $D^{n}$ is the closed unit ball in $\mathbf{R}^{n}$, and where $f$ is a continuous function from the boundary $S^{n-1}$ of $D^{n}$ to $Y$. Note that $f$ induces a continuous function $g: D^{n} \rightarrow X$ which agrees with $f$ on $S^{n-1}$ and is a homeomorphism from $D^{n} \backslash S^{n-1}$ to $X \backslash Y$. Pulling everything back via $f$ or $g$ as appropriate, we further reduce to the case $Y=S^{n-1}, X=D^{n}$, and the attaching map is the identity. Since $D^{n}$ is contractible, we may assume all bundles are trivial.

For part (1), let $\operatorname{rank}(E)=r$, so that $E=D^{n} \times \mathbf{C}^{r}$. First $\operatorname{suppose} \operatorname{rank}(F)=1$, so that $F=D^{n} \times \mathbf{C}$. In this case, $a_{0}$ is determined by a continuous function (section) $\xi_{0}: S^{n-1} \rightarrow \mathbf{C}^{r} \backslash\{0\}$. Now $\mathbf{C}^{r} \backslash\{0\}$ is homotopy equivalent to $S^{2 r-1}$, and the dimension hypotheses imply that $n-1<2 r-1$, so $\xi_{0}$ extends to a continuous function (section) $\xi: D^{n} \rightarrow \mathbf{C}^{r} \backslash\{0\}$. This section gives the required isomorphism of $F$ with a subbundle of $E$.

We now do the general case of part (1) by induction on $s=\operatorname{rank}(F)$. Suppose the result is known for $F$ of rank $s$, and the actual rank of $F$ is $s+1$. Write $F=F_{0} \oplus$ $\left(D^{n} \times \mathbf{C}\right)$. Use the induction hypothesis to extend $\left.a_{0}\right|_{\left(\left.F_{0}\right|_{S^{n-1}}\right)}$ to an isomorphism $c$ of $F_{0}$ with a subbundle of $E$ such that $\left.c\right|_{S^{n-1}}=\left.a_{0}\right|_{\left(\left.F_{0}\right|_{S^{n-1}}\right)}$. Next, use the rank one case to extend $\left.a_{0}\right|_{S^{n-1} \times \mathbf{C}}$ to an isomorphism $d$ of $D^{n} \times \mathbf{C}$ with a subbundle of the orthogonal complement in $E$ of $c\left(F_{0}\right)$, such that $\left.d\right|_{S^{n-1}}=\left.a_{0}\right|_{S^{n-1} \times \mathbf{C}}$. Then set $a=c+d$.

Next we do (2). Without loss of generality, $E_{1}=E_{2}=D^{n} \times \mathbf{C}^{r}$ and $F=D^{n} \times \mathbf{C}^{s}$ for suitable $r$ and $s$. The given maps can then be thought of as $a_{0}: S^{n-1} \rightarrow \mathrm{GL}_{r}(\mathbf{C})$, $b: D^{n} \rightarrow \mathrm{GL}_{r+s}(\mathbf{C})$, and $c_{t}^{(0)}: S^{n-1} \rightarrow \mathrm{GL}_{r+s}(\mathbf{C})$, such that $t \mapsto c_{t}^{(0)}$ is a homotopy from $a_{0} \oplus 1$ to $\left.b\right|_{S^{n-1}}$. In particular, $a_{0}$ defines a class in $\pi_{n-1}\left(\mathrm{GL}_{r}(\mathbf{C})\right)$ whose image in $\pi_{n-1}\left(\mathrm{GL}_{r+s}(\mathbf{C})\right)$ is zero. Using the fact that the unitary groups are deformation retracts of the corresponding invertible groups, Theorem 8.4.1 of [16] implies that $\pi_{n-1}\left(\mathrm{GL}_{r}(\mathbf{C})\right) \rightarrow \pi_{n-1}\left(\mathrm{GL}_{r+s}(\mathbf{C})\right)$ is an isomorphism when $n-1 \leq 2 r-1$. This inequality is satisfied in our case, so $a_{0}$ is null homotopic. Therefore, there is a continuous function $\widetilde{a}: D^{n} \rightarrow \mathrm{GL}_{r}(\mathbf{C})$ which extends $a_{0}$.

Let $h:[0,1] \times D^{n} \rightarrow D^{n+1}$ be a homeomorphism. Then, with

$$
S=\left(\{0,1\} \times D^{n}\right) \cup\left([0,1] \times S^{n-1}\right),
$$

we have $h(S)=S^{n}$. Let $\widetilde{v}: S^{n} \rightarrow \mathrm{GL}_{r+s}(\mathbf{C})$ be the function corresponding to the function on $S$ given by $\widetilde{a}(x) \oplus 1$ on $\{0\} \times D^{n}$, by $b(x)$ on $\{1\} \times D^{n}$, and by $c_{t}^{(0)}(x)$ on $[0,1] \times S^{n-1}$. Let $\eta=[\widetilde{v}] \in \pi_{n}\left(\mathrm{GL}_{r+s}(\mathbf{C})\right)$. Using Theorem 8.4.1 of [16] in the same manner as above, we find that $\pi_{n}\left(\mathrm{GL}_{r}(\mathbf{C})\right) \rightarrow \pi_{n}\left(\mathrm{GL}_{r+s}(\mathbf{C})\right)$ is surjective. So there is a function $w: S \rightarrow \mathrm{GL}_{r}(\mathbf{C})$ such that $[w] \in \pi_{n}\left(\mathrm{GL}_{r}(\mathbf{C})\right)$ has image $-\eta \in$ $\pi_{n}\left(\mathrm{GL}_{r+s}(\mathbf{C})\right)$. A simple deformation argument allows us to require in addition that $w(x)=1$ for $x \in\left(\{1\} \times D^{n}\right) \cup\left([0,1] \times S^{n-1}\right)$. We have $[w \oplus 1]+[\widetilde{v}]=[(w \oplus 1) \widetilde{v}]$ in $\pi_{n}\left(\mathrm{GL}_{r+s}(\mathbf{C})\right)$ (see Corollary 1.6.10 of [40]), whence $(w \oplus 1) \widetilde{v}$ is null homotopic. Define $a=w \widetilde{a}$. Since $w=1$ on $\{0\} \times S^{n-1}$, we also have $\left.a\right|_{S^{n-1}}=a_{0}$. Further define $v: S \rightarrow \mathrm{GL}_{r+s}(\mathbf{C})$ by substituting $a$ for $\widetilde{a}$ in the definition of $\widetilde{v}$. Then $v=(w \oplus 1) \widetilde{v}$ is null homotopic, and hence extends continuously to a function $(t, x) \mapsto c_{t}(x)$ from $[0,1] \times D^{n}$ to $\mathrm{GL}_{r+s}(\mathbf{C})$. Note that

$$
\left.c_{t}\right|_{S^{n-1}}=\left.v\right|_{\{t\} \times S^{n-1}}=\left.\widetilde{v}\right|_{\{t\} \times S^{n-1}}=\left.c_{t}^{(0)}\right|_{S^{n-1}} .
$$

So $t \mapsto c_{t}$ is the required homotopy. 
Proposition 4.2. Let $X$ be a compact Hausdorff space with covering dimension $\operatorname{dim}(X) \leq d$, and let $Y \subset X$ be closed.

(1) Let $p, q \in C\left(X, M_{n}\right)$ be projections. Suppose $\operatorname{rank}(p(x))-\operatorname{rank}(q(x)) \geq$ $\frac{1}{2}(d-1)$ for all $x \in X$. Let $s_{0} \in C\left(Y, M_{n}\right)$ satisfy $s_{0}^{*} s_{0}=\left.q\right|_{Y}$ and $s_{0} s_{0}^{*} \leq\left. p\right|_{Y}$. Then there is $s \in C\left(X, M_{n}\right)$ such that

$$
s^{*} s=q, \quad s s^{*} \leq p, \quad \text { and }\left.\quad s\right|_{Y}=s_{0} .
$$

(2) Let $p_{1}, p_{2}, q_{1}, q_{2} \in C\left(X, M_{n}\right)$ be projections, and let $u, s \in C\left(X, M_{n}\right)$. Assume that $\operatorname{rank}\left(p_{j}(x)\right) \geq \frac{1}{2} d$ for all $x$, that $p_{j} \perp q_{j}$, and that

$$
s^{*} s=q_{1}, \quad s s^{*}=q_{2}, \quad u^{*} u=p_{1}+q_{1}, \quad \text { and } \quad u u^{*}=p_{2}+q_{2} .
$$

Further, let $v_{0} \in C\left(Y, M_{n}\right)$ satisfy $v_{0}^{*} v_{0}=\left.p_{1}\right|_{Y}$ and $v_{0} v_{0}^{*}=\left.p_{2}\right|_{Y}$, and let $t \mapsto w_{t}^{(0)}$ be a continuous path of partial isometries in $C\left(Y, M_{n}\right)$ such that

$$
\left(w_{t}^{(0)}\right)^{*} w_{t}^{(0)}=\left.\left(p_{1}+q_{1}\right)\right|_{Y} \quad \text { and } \quad w_{t}^{(0)}\left(w_{t}^{(0)}\right)^{*}=\left.\left(p_{2}+q_{2}\right)\right|_{Y},
$$

and

$$
w_{0}^{(0)}=\left.u\right|_{Y} \quad \text { and } \quad w_{1}^{(0)}=v_{0}+\left.s\right|_{Y} .
$$

Then there is $v \in C\left(X, M_{n}\right)$ such that

$$
v^{*} v=p_{1}, \quad v v^{*}=p_{2}, \quad \text { and }\left.\quad v\right|_{Y}=v_{0},
$$

and a continuous path $t \mapsto w_{t}$ of partial isometries in $C\left(X, M_{n}\right)$ such that

$$
w_{t}^{*} w_{t}=p_{1}+q_{1}, \quad w_{t} w_{t}^{*}=p_{2}+q_{2}, \quad w_{0}=u, \quad w_{1}=v+s, \quad \text { and }\left.\quad w_{t}\right|_{Y}=w_{t}^{(0)} .
$$

Proof. We reduce this to Proposition 4.1 in a number of steps.

The first reduction is to the case that all the projections involved have constant ranks. In (1), this means that $\operatorname{rank}(p(x))$ and $\operatorname{rank}(q(x))$ are independent of $x$; similarly for (2). This is accomplished by writing $X$ as a finite disjoint union of closed and open subsets on which all the ranks are constant.

Second, we reduce part (2) to the situation $q_{1}=q_{2}=s=q$. To do this, first replace (without renaming) $M_{n}$ by $M_{2 n}$, and replace each of $p_{j}, q_{j}, u, s, v_{0}$, and $t \mapsto w_{t}^{(0)}$ by its block diagonal direct sum with the $n \times n$ zero matrix, namely $p_{j} \oplus 0, q_{j} \oplus 0$, etc. Validity of the conclusion in the new situation is equivalent to its validity in the old. Now set

$$
z=\left(\begin{array}{cc}
s & 1-s s^{*} \\
1-s^{*} s & s^{*}
\end{array}\right)
$$

which is a unitary satisfying $z\left(q_{1} \oplus 0\right) z^{*}=q_{2} \oplus 0$. Define

$$
\widetilde{q}=q_{2} \oplus 0=z\left(q_{1} \oplus 0\right) z^{*}=(s \oplus 0) z^{*}, \quad \widetilde{p}_{1}=z\left(p_{1} \oplus 0\right) z^{*}, \quad \text { and } \quad \widetilde{p}_{2}=p_{2} \oplus 0,
$$
and

$$
\widetilde{u}=(u \oplus 0) z^{*}, \quad \widetilde{v}_{0}=\left(v_{0} \oplus 0\right) z^{*}, \quad \text { and } \quad \widetilde{w}_{t}^{(0)}=\left(w_{t}^{(0)} \oplus 0\right) z^{*} .
$$

Assuming the proposition holds for this case (with $\widetilde{q}_{1}=\widetilde{q}_{2}=\widetilde{s}=\widetilde{q}$ ), let $\widetilde{v}$ and $\widetilde{w}_{t}$ be the resulting partial isometry and homotopy. Then the conclusion (2) as stated holds with

$$
v=\left(\begin{array}{cc}
1 & 0 \\
0 & 0
\end{array}\right) \widetilde{v} z\left(\begin{array}{cc}
1 & 0 \\
0 & 0
\end{array}\right) \quad \text { and } \quad w_{t}=\left(\begin{array}{cc}
1 & 0 \\
0 & 0
\end{array}\right) \widetilde{w}_{t} z\left(\begin{array}{cc}
1 & 0 \\
0 & 0
\end{array}\right),
$$

regarded as elements of $C\left(X, M_{n}\right)$.

In preparation for our next reduction, we prove the following approximation result. There is an absolute constant $\varepsilon>0$ such that, if the hypotheses of (1) 
are satisfied, and there are a compact Hausdorff space $\tilde{X}$, a closed subset $\widetilde{Y} \subset \widetilde{X}$, a continuous function $h: X \rightarrow \widetilde{X}$ with $h(Y)=\widetilde{Y}$, and $\widetilde{p}, \widetilde{q} \in C\left(\widetilde{X}, M_{n}\right)$ and $\widetilde{s}_{0} \in C\left(\tilde{Y}, M_{n}\right)$ for which the hypotheses and conclusion hold, moreover, with

$$
\|\widetilde{p} \circ h-p\|<\varepsilon, \quad\|\widetilde{q} \circ h-q\|<\varepsilon, \quad \text { and } \quad\left\|\widetilde{s}_{0} \circ h-s_{0}\right\|<\varepsilon,
$$

then the conclusion of (1) holds for $p, q$, and $s_{0}$. The analogous statement is true for part (2), in the case $q_{1}=q_{2}=s=q$.

To see this in part $(1)$, let $\widetilde{s} \in C\left(\widetilde{X}, M_{n}\right)$ be the partial isometry obtained from the assumption that (1) holds for $\widetilde{X}$ and $\widetilde{Y}$. Let $a \in C\left(X, M_{n}\right)$ be an arbitrary extension of $s_{0}$. Choose a neighborhood $U$ of $Y$ such that $\|\widetilde{s} \circ h(x)-a(x)\|<2 \varepsilon$ for $x \in U$. (This norm is less than $\varepsilon$ on $Y$.) Choose a continuous function $f: X \rightarrow[0,1]$ which is equal to 1 on $Y$ and vanishes outside $U$. Define

$b(x)=p(x)[f(x) a(x)+(1-f(x)) \widetilde{s} \circ h(x)] q(x) \quad$ and $\quad s(x)=b(x)\left[b(x)^{*} b(x)\right]^{-1 / 2}$ (functional calculus in $q(x) M_{n} q(x)$ ). It is easily seen that if $\varepsilon$ is small enough, then $s$ is the required partial isometry.

The proof for (2) is similar. The formulas are as follows. Let $a \in C\left(X, M_{n}\right)$ be an arbitrary extension of $v_{0}$. Let $f: X \rightarrow[0,1]$ be equal to 1 on $Y$ and vanish outside a suitable neighborhood of $Y$, and set

$$
b(x)=p_{2}(x)[f(x) a(x)+(1-f(x)) \widetilde{v} \circ h(x)] p_{1}(x)
$$

and

$$
v(x)=b(x)\left[b(x)^{*} b(x)\right]^{-1 / 2}
$$

(functional calculus in $\left.p_{1}(x) M_{n} p_{1}(x)\right)$. Then let $(t, x) \mapsto c(t, x)$ be an element of $C\left([0,1] \times X, M_{n}\right)$ which extends the function

$$
(t, x) \mapsto \begin{cases}w_{t}^{(0)}(x), & t \in[0,1], x \in Y, \\ v(x)+q(x), & t=1, x \in X, \\ 1, & t=0, x \in X .\end{cases}
$$

Let $g:[0,1] \times X \rightarrow[0,1]$ be equal to 1 on $([0,1] \times Y) \cup(\{0,1\} \times X)$ and vanish outside a suitable neighborhood of this set, and set

$$
d(t, x)=\left[p_{2}(x)+q(x)\right]\left[g(t, x) c(t, x)+(1-g(t, x)) \widetilde{w}_{t} \circ h(x)\right]\left[p_{1}(x)+q(x)\right]
$$

and

$$
w_{t}(x)=d(t, x)\left[d(t, x)^{*} d(t, x)\right]^{-1 / 2}
$$

(functional calculus in $\left[p_{1}(x)+q(x)\right] M_{n}\left[p_{1}(x)+q(x)\right]$ ).

It is clear from this approximation result that if $X=\lim X_{\alpha}$, and the proposition holds for all pairs $\left(X_{\alpha}, Y\right)$ with $Y \subset X_{\alpha}$ closed, then it holds for all pairs $(X, Y)$ with $Y \subset X$ closed. (The function $h$ will be the map to a suitable $X_{\alpha}$.) Our third reduction is from $X$ a compact space of dimension at most $d$ to $X$ a compact metric space of dimension at most $d$. This follows because $\operatorname{dim}(X) \leq d \operatorname{implies} X \cong \lim X_{\alpha}$ with $X_{\alpha}$ compact metric and $\operatorname{dim}\left(X_{\alpha}\right) \leq d$ (Theorem 3.3.7 of [13]). For the fourth reduction, apply Theorem 1.13 .5 of 13 . (every compact space of dimension at most $d$ is the inverse limit of finite simplicial complexes dimension at most $d$ ) to reduce to the case in which $X$ is a finite simplicial complex (but still $Y$ is an arbitrary closed subset of $X$ ).

Our fifth reduction is to the situation in which $Y$ is a subcomplex. An argument very similar to the approximation argument above allows us to assume that $s_{0}$ in part (1), and $v_{0}$ and $w_{t}^{(0)}$ in part (2), are actually defined on an open set $U \supset Y$. Let 
$\varepsilon=\operatorname{dist}(Y, X \backslash U)>0$. By repeated barycentric subdivision, we may assume that all the simplexes in $X$ have diameter less than $\frac{1}{2} \varepsilon$. (See the proofs of Corollary 3.3.13 and Theorem 3.3.14 of 40.) Let $\tilde{Y}$ be the closed subset of $X$ given as the union of all faces (of any dimension) of all simplexes in $X$ which intersect $Y$. The conclusion is now proved by applying the proposition for the case of a subcomplex to $\left.s_{0}\right|_{\widetilde{Y}}$ in part (1), and to $\left.v_{0}\right|_{\widetilde{Y}}$ and $\left.w_{t}^{(0)}\right|_{\widetilde{Y}}$ in part (2).

It remains only to prove the result for the case that $Y$ is a subcomplex of $X$. For (1), apply Proposition 4.1(1) with $E$ and $F$ the vector bundles determined by $E_{x}=p(x) \mathbf{C}^{n}$ and $F_{x}=q(x) \mathbf{C}^{n}$, and with $a_{0}=s_{0}$. (This is Swan's Theorem; see Theorem 2 of [41.) The resulting isomorphism $a$ of $F$ with a subbundle of $E$ is an element of $C\left(X, M_{n}\right)$ such that $a^{*} a$ is invertible in $q C\left(X, M_{n}\right) q$, so we can set $s=a\left(a^{*} a\right)^{-1 / 2}$. The same application of Swan's Theorem and unitarization reduces part (2) (as reduced in the first reduction of this proof) to part (2) of Proposition 4.1.

Now we can prove the analogs of Theorem 2.5(b) and (c) of [15. (The analog of Theorem 2.5(a) of [15] does not make sense in this context, because there might not be any rank one projections in a recursive subhomogeneous algebra.)

We follow Definition 5.1.1 of [2], and write $M_{\infty}(A)$ for the algebraic direct limit $\lim _{n} M_{n}(A)$ under the maps $a \mapsto a \oplus 0$.

In part (2) of the following proposition, we would really like to allow $\operatorname{rank}\left(\mathrm{ev}_{x}(e)\right)$ to be either zero or greater than $\frac{1}{2} d$. We have not been able to decide whether this is possible; there might be a topological obstruction.

Proposition 4.3. Let $A$ be a recursive subhomogeneous algebra with total space $X$, and with topological dimension function $d: X \rightarrow \mathbf{N} \cup\{0\}$ (as in Definition 1.2). Let $p, e, f, q \in M_{\infty}(A)$ be projections.

(1) If for every $x \in X$, either

$$
q(x)=0 \quad \text { or } \operatorname{rank}\left(\mathrm{ev}_{x}(p)\right)-\operatorname{rank}\left(\mathrm{ev}_{x}(q)\right) \geq \frac{1}{2}[d(x)-1],
$$

then $q \precsim p$.

(2) If $e \oplus q \sim f \oplus q$ and $\operatorname{rank}\left(\operatorname{ev}_{x}(e)\right) \geq \frac{1}{2} d(x)$ for every $x \in X$, then $e \sim f$.

Proof. We may assume everything is in $M_{n}(A)$ for some $n$. Since $M_{n}(A)$ is also a recursive subhomogeneous algebra with the same base spaces (see Lemma 1.12) and hence the same topological dimension function, we need only consider projections in $A$.

For (1), we first carry out a reduction. Adopt the notation of Definition 1.2, Let $q_{k}$ be the image of $q$ in $C\left(X_{k}, M_{n(k)}\right)$. Define

$$
Y_{k}=\left\{x \in X_{k}: q_{k}(x)=0\right\} \text { and } Z_{k}=Y_{k} \backslash X_{k} .
$$

Applying Lemma 1.9, we construct a new decomposition for $A$ using the spaces $Y_{k}$ and $Z_{k}$ instead of $X_{k}$. It has length at most $2 l+1$, the same total space, and the new topological dimension function is dominated by the old one, $\operatorname{since} \operatorname{dim}\left(X_{k}\right)=$ $\max \left(\operatorname{dim}\left(Y_{k}\right), \operatorname{dim}\left(Z_{k}\right)\right)$. Using the old notation for this new decomposition, it has the property that, for every $k$, either $q_{k}=0$ or $\operatorname{rank}\left(q_{k}(x)\right) \geq \frac{1}{2}[d(x)-1]$ for all $x \in X_{k}$.

We now prove the result by induction on the length $l$ of a decomposition with this property. If the length is zero, then $A=C\left(X, M_{n}\right)$ for some $X$ and $n$. In this 
case, if $q=0$, then the conclusion is trivial, while if $\operatorname{rank}(q(x)) \geq \frac{1}{2}[\operatorname{dim}(X)-1]$ for all $x \in X$, then the conclusion follows from the case $Y=\varnothing$ of Proposition $4.2(1)$.

Now suppose the result is known for length $l$. Let $A=B \oplus_{C\left(X^{(0)}, M_{n}\right)} C\left(X, M_{n}\right)$, with $\varphi: B \rightarrow C\left(X^{(0)}, M_{n}\right)$ unital and $\rho: C\left(X, M_{n}\right) \rightarrow C\left(X^{(0)}, M_{n}\right)$ the restriction map, where $B$ is a recursive subhomogeneous algebra of length $l$. Write $p=\left(p_{1}, p_{2}\right)$ and $q=\left(q_{1}, q_{2}\right)$ with $p_{1}, q_{1} \in B$ and $p_{2}, q_{2} \in C\left(X, M_{n}\right)$. By the induction assumption, there is $s_{1} \in B$ such that $s_{1}^{*} s_{1}=q_{1}$ and $s_{1} s_{1}^{*} \leq p_{1}$.

If $q_{2}=0$, then $\varphi\left(s_{1}\right)=0$. Therefore, $s=\left(s_{1}, 0\right)$ satisfies $s^{*} s=q$ and $s s^{*} \leq p$, so $q \precsim p$ as desired. Otherwise, $\operatorname{rank}\left(q_{2}(x)\right) \geq \frac{1}{2}[\operatorname{dim}(X)-1]$ for all $x \in X$. Apply Proposition $4.2(1)$ to $p_{2}, q_{2}, X^{(0)} \subset X$, and with the partial isometry $\varphi\left(s_{1}\right) \in$ $C\left(X^{(0)}, M_{n}\right)$. Let $s_{2}$ be the resulting partial isometry. Then $s=\left(s_{1}, s_{2}\right)$ is a partial isometry implementing the relation $q \precsim p$. This completes the induction, and proves (1).

For (2), we also use induction on the length. We actually prove a stronger result (needed for the induction to work): given a partial isometry $u$ with $u^{*} u=e+q$ and $u u^{*}=f+q$, there is a partial isometry $s$ with $s^{*} s=e, s s^{*}=f$, and $s+q$ homotopic to $u$ among partial isometries from $e+q$ to $f+q$.

In the initial step (length 0 ), the conclusion follows from the case $Y=\varnothing$ of Proposition $4.2(2)$. In the induction step, use the same notation as in the induction step for (1), and in addition let $e=\left(e_{1}, e_{2}\right), f=\left(f_{1}, f_{2}\right)$, and $u=\left(u_{1}, u_{2}\right)$. The induction assumption provides a partial isometry $s_{1} \in B$ such that $s_{1}^{*} s_{1}=e_{1}$, $s_{1} s_{1}^{*}=f$, and there is a homotopy $t \mapsto w_{1}^{(t)}$ from $s_{1}+q_{1}$ to $u_{1}$ in the set of partial isometries from $e_{1}+q_{1}$ to $f_{1}+q_{1}$. Use Proposition $4.2(2)$ on $X^{(0)} \subset X$ to find $s_{2} \in C\left(X, M_{n}\right)$ and $t \mapsto w_{2}^{(t)} \in C\left(X, M_{n}\right)$, extending $\varphi\left(s_{1}\right)$ and $t \mapsto \varphi\left(w_{2}^{(t)}\right)$ respectively, such that $s_{2}^{*} s_{2}=e_{2}, s_{2} s_{2}^{*}=f$, and $t \mapsto w_{2}^{(t)}$ is a homotopy from $s_{2}+q_{2}$ to $u_{2}$ in the set of partial isometries from $e_{2}+q_{2}$ to $f_{2}+q_{2}$. Define

$$
w^{(t)}=\left(w_{1}^{(t)}, w_{2}^{(t)}\right) \text { and } s=\left(s_{1}, s_{2}\right)
$$

Then $s$ is a partial isometry from $e$ to $f$, and $t \mapsto w^{(t)}$ is a homotopy from $s+q$ to $u$ in the set of partial isometries from $e+q$ to $f+q$. This completes the induction, and the proof of $(2)$.

The following proposition is an analog of Proposition 4.3 for unitaries instead of projections.

Proposition 4.4. Let $A$ be a recursive subhomogeneous algebra with total space $X$, and with topological dimension function $d: X \rightarrow \mathbf{N} \cup\{0\}$ (as in Definition [1.2). Let $p, q \in M_{\infty}(A)$ be projections with $p \leq q$.

(1) If $\operatorname{rank}\left(\operatorname{ev}_{x}(p)\right) \geq \frac{1}{2} d(x)$ for all $x \in X$, and $u \in U\left(q M_{\infty}(A) q\right)$, then there exists $v \in U\left(p M_{\infty}(A) p\right)$ such that $v+(q-p)$ is homotopic to $u$ in $U\left(q M_{\infty}(A) q\right)$.

(2) If $\operatorname{rank}\left(\operatorname{ev}_{x}(p)\right) \geq \frac{1}{2}[d(x)+1]$ for all $x \in X$, and $v^{(0)}, v^{(1)} \in U\left(p M_{\infty}(A) p\right)$ are unitaries such that $v^{(0)}+(q-p)$ is homotopic to $v^{(1)}+(q-p)$ in $U\left(q M_{\infty}(A) q\right)$, then $v^{(0)}$ is homotopic to $v^{(1)}$ in $U\left(p M_{\infty}(A) p\right)$.

Proof. As in the proof of Proposition 4.3, we may assume $q \in A$.

We prove the result by induction on the length $l$ of a decomposition. We describe only the induction steps, since the initial steps differ only in that the subset $Y$ in Proposition 4.2 is taken to be empty (in (1)) or just $\{0,1\} \times X$ (in (2)). 
So suppose part (1) is known for recursive subhomogeneous algebras of length l. Let $A=B \oplus_{C\left(X^{(0)}, M_{n}\right)} C\left(X, M_{n}\right)$, with $\varphi: B \rightarrow C\left(X^{(0)}, M_{n}\right)$ unital and $\rho: C\left(X, M_{n}\right) \rightarrow C\left(X^{(0)}, M_{n}\right)$ the restriction map, where $B$ is a recursive subhomogeneous algebra of length $l$. Write $p=\left(p_{1}, p_{2}\right), q=\left(q_{1}, q_{2}\right)$, and $u=\left(u_{1}, u_{2}\right)$, with $p_{1}, q_{1}, u_{1} \in B$ and $p_{2}, q_{2}, u_{2} \in C\left(X, M_{n}\right)$. By the induction assumption, there are $v_{1} \in U\left(p_{1} B p_{1}\right)$ and $t \mapsto w_{1}^{(t)} \in U\left(q_{1} B q_{1}\right)$ such that $w_{1}^{(0)}=u_{1}$ and $w_{1}^{(1)}=v_{1}+\left(q_{1}-p_{1}\right)$. Apply Proposition $4.2(2)$ to $X^{(0)} \subset X$, with the $p_{i}$ there both being $p_{2}$, the $q_{i}$ both being $q_{2}-p_{2}, v_{0}$ being $\varphi\left(v_{1}\right), s$ being $q_{2}-p_{1}$, and the homotopy being $t \mapsto \varphi\left(w_{1}^{(t)}\right)$. Let $v_{2}$ be the resulting element of $U\left(p_{2} C\left(X, M_{n}\right) p_{2}\right)$ and let $t \mapsto w_{2}^{(t)} \in U\left(q_{2} C\left(X, M_{n}\right) q_{2}\right)$ be the resulting homotopy. The elements $v=\left(v_{1}, v_{2}\right)$ and $w^{(t)}=\left(w_{1}^{(t)}, w_{2}^{(t)}\right)$ prove the induction step.

For part (2), we use analogous notation, and we assume for the induction step that we are given homotopies $t \mapsto c^{(t)}$ from $v^{(0)}+(q-p)$ to $v^{(1)}+(q-p)$ in $U(q A q)$ and $t \mapsto v_{1}^{(t)}$ from $v_{1}^{(0)}$ to $v_{1}^{(1)}$ in $U\left(p_{1} B p_{1}\right)$. We regard the homotopies as elements of the recursive subhomogeneous algebra $C([0,1], A)$ with its obvious corresponding decomposition (see Lemma 1.14), and we require as part of the induction hypothesis that there be a homotopy $\left(t_{1}, t_{2}\right) \mapsto w_{1}^{\left(t_{1}, t_{2}\right)} \in U\left(q_{1} B q_{1}\right)$ of elements of $C([0,1], B)$ such that

$$
w_{1}^{\left(0, t_{2}\right)}=v_{1}^{\left(t_{2}\right)}+\left(q_{1}-p_{1}\right) \quad \text { and } \quad w_{1}^{\left(1, t_{2}\right)}=c_{1}^{\left(t_{2}\right)}
$$

for all $t_{2}$, and

$$
w_{1}^{\left(t_{1}, 0\right)}=v_{1}^{(0)}+\left(q_{1}-p_{1}\right) \quad \text { and } \quad w_{1}^{\left(t_{1}, 1\right)}=v_{1}^{(1)}+\left(q_{1}-p_{1}\right)
$$

for all $t_{1}$. We apply Proposition $4.2(2)$ to

$$
\left([0,1] \times X^{(0)}\right) \cup(\{0,1\} \times X) \subset[0,1] \times X,
$$

with the $p_{i}$ there both being $(t, x) \mapsto p_{2}(x)$, with the $q_{i}$ both being $(t, x) \mapsto q_{2}(x)-$ $p_{2}(x)$, with $v_{0}$ there being $(t, x) \mapsto \varphi\left(v_{1}^{(t)}\right)(x)$ on $[0,1] \times X^{(0)}$ and $v_{2}^{(i)}(x)$ on $\{i\} \times X$, with $u$ there being $(t, x) \mapsto c_{2}^{(t)}(x)$, with $s$ there being $(t, x) \mapsto q_{2}(x)-p_{2}(x)$, and with $(t, y) \mapsto w_{t}^{(0)}(y)$ being

$$
\left(t_{1}, t_{2}, x\right) \mapsto \begin{cases}\varphi\left(w_{1}^{\left(t_{1}, t_{2}\right)}\right)(x), & x \in X^{(0)}, \\ v_{2}^{\left(t_{2}\right)}(x)+\left(q_{2}(x)-p_{2}(x)\right), & t_{2} \in\{0,1\} .\end{cases}
$$

(Here $t_{1}=t$ and $\left(t_{2}, x\right)=y$.) Note that $\operatorname{dim}([0,1] \times X) \leq \operatorname{dim}(X)+1$ by Proposition 3.2.6 of 31]. We then obtain a unitary $(t, x) \mapsto v_{2}^{(t)}(x)$ in $q_{2}\left[C\left([0,1] \times X, M_{n}\right)\right] q_{2}$ extending $(t, x) \mapsto \varphi\left(v_{1}^{(t)}\right)(x)$ and agreeing at $t=0$ and $t=1$ with $v_{2}^{(0)}$ and $v_{2}^{(1)}$ as already defined, and also a homotopy $\left(t_{1}, t_{2}\right) \mapsto w_{2}^{\left(t_{1}, t_{2}\right)} \in U\left(q_{2} C\left(X, M_{n}\right) q_{2}\right)$ from $t_{2} \mapsto v_{2}^{\left(t_{2}\right)}+\left(q_{2}-p_{2}\right)$ to $t_{2} \mapsto c_{2}^{\left(t_{2}\right)}$ extending the given homotopy, in particular, satisfying $w_{2}^{\left(t_{1}, t_{2}\right)}(x)=v_{2}^{\left(t_{2}\right)}(x)+\left(q_{2}(x)-p_{2}(x)\right)$ for $x \in X, t_{1} \in[0,1]$, and $t_{2} \in\{0,1\}$. The induction step is completed by taking

$$
v^{(t)}=\left(v_{1}^{(t)}, v_{2}^{(t)}\right) \quad \text { and } \quad w^{\left(t_{1}, t_{2}\right)}=\left(w_{1}^{\left(t_{1}, t_{2}\right)}, w_{2}^{\left(t_{1}, t_{2}\right)}\right) .
$$

One readily checks that the properties assumed in $B$ have been extended to $A$.

Although the primary intended application of the results of this section is to direct limits of recursive subhomogeneous algebras (see [35] and [36]), there are immediate interesting consequences for type $1 \mathrm{C}^{*}$-algebras. As before Theorem 2.12, 
we let $\operatorname{Prim}_{n}(A)$ denote the subspace of $\operatorname{Prim}(A)$ consisting of the kernels of $n$ dimensional representations of $A$.

Theorem 4.5. Let $A$ be a separable unital $C^{*}$-algebra. Suppose that there is $N \in \mathbf{N}$ such that all irreducible representations of $A$ have dimension at most $N$. Let $p, q \in$ $M_{\infty}(A)$ be projections. Suppose that for every $n$ and every irreducible representation $\pi$ of $A$ of dimension $n$, we have

$$
\operatorname{rank}(\pi(p))-\operatorname{rank}(\pi(q)) \geq \frac{1}{2}\left[\operatorname{dim}\left(\operatorname{Prim}_{n}(A)\right)-1\right] .
$$

Then $q \precsim p$.

Proof. Lemma2.5 and Proposition 2.9 allow us to apply Proposition 2.13. Using the standard notation (see Definition 1.2) for the resulting recursive subhomogeneous decomposition, we then have:

(1) $n(0) \leq n(1) \leq \cdots \leq n(l)$.

(2) $\operatorname{dim}\left(X_{k}\right) \leq \operatorname{dim}\left(\operatorname{Prim}_{n(k)}(A)\right)$ for $0 \leq k \leq l$.

(3) $X_{k} \backslash X_{k}^{(0)}$ is dense in $X_{k}$ for $1 \leq k \leq l$.

Let $X$ be the total space, and let $d: X \rightarrow \mathbf{N} \cup\{0\}$ be the topological dimension function (as in Definition 1.2). Take $X_{0}^{(0)}=\varnothing$. By Lemma 2.1, the evaluations $\mathrm{ev}_{x}$, for $x \in X_{k} \backslash X_{k}^{(0)}$, are irreducible. Therefore, for $x \in X_{k} \backslash X_{k}^{(0)}$, we have

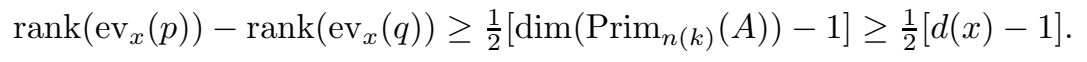

By continuity, this inequality is valid for all $x \in \overline{X_{k} \backslash X_{k}^{(0)}}=X_{k}$. Now Proposition 4.3(1) implies that $q \precsim p$.

Theorem 4.6. Let $A$ be a separable unital $C^{*}$-algebra. Suppose that there is $N \in$ $\mathbf{N}$ such that all irreducible representations of $A$ have dimension at most $N$. Let $e, f, q \in M_{\infty}(A)$ be projections such that $e \oplus q \sim f \oplus q$. Suppose that for every $n$ and every irreducible representation $\pi$ of $A$ of dimension $n$, we have

$$
\operatorname{rank}(\pi(e)) \geq \frac{1}{2} \operatorname{dim}\left(\operatorname{Prim}_{n}(A)\right) .
$$

Then $e \sim f$.

Proof. The proof is the same as that of Theorem 4.5, except that in the last step we apply Proposition 4.3(2).

Theorem 4.7. Let $A$ be a separable unital $C^{*}$-algebra. Suppose that there is $N \in \mathbf{N}$ such that all irreducible representations of $A$ have dimension at most $N$, and suppose that $\operatorname{dim}\left(\operatorname{Prim}_{n}(A)\right) \leq 2 n-1$ for all $n$. Then the natural map $U(A) / U_{0}(A) \rightarrow K_{1}(A)$ is an isomorphism.

Proof. We first prove injectivity. Thus, let $u, v \in A$ be unitary, and suppose that $[u]=[v]$ in $K_{1}(A)$; we must show that $u$ can be connected to $v$ by a path in $U(A)$. By assumption, there is $n$ such that $u \oplus 1$ can be connected to $v \oplus 1$ in $U\left(M_{n}(A)\right)$. As in the proof of Theorem 4.5, there is a recursive subhomogeneous decomposition for $A$ with total space $X$ and topological dimension function $d: X \rightarrow \mathbf{N} \cup\{0\}$ such that $\operatorname{rank}\left(\operatorname{ev}_{x}\left(1_{A}\right)\right) \geq \frac{1}{2}[d(x)+1]$ for every $x \in X$. Apply Proposition $4.4(2)$ with $p=1_{A}$ and $q=1_{M_{n}(A)}$.

Surjectivity is proved in the same way, using instead Proposition 4.4(1) at the end. 
If $A=C\left(X, M_{n}\right)$ with $\operatorname{dim}(X) \leq 2 n-1$, then one can deduce the conclusion of Theorem 4.7 from stable rank considerations. Specifically, Proposition 1.7 of [38] gives $\operatorname{tsr}(C(X)) \leq n$, and it then follows from Theorem 2.10 of [39] that

$$
U\left(C\left(X, M_{n}\right)\right) / U_{0}\left(C\left(X, M_{n}\right)\right) \rightarrow K_{1}(A)
$$

is an isomorphism. However, it is not clear how to obtain the general case of Theorem 4.7 from stable rank considerations. As just one of several difficulties, we note that, with $X$ and $n$ as above, Theorem 6.1 of 38 gives $\operatorname{tsr}\left(C\left(X, M_{n}\right)\right)=2$ unless $\operatorname{dim}(X) \leq 1$. Applying Theorem 4.3 of 38 , we see that an algebra $A$ as in the hypotheses of Theorem 4.7 generally has stable rank at least 2 , so that we can't apply Theorem 2.10 of [39].

\section{REFERENCES}

[1] C. A. Akemann, G. K. Pedersen, and J. Tomiyama, Multipliers of $C^{*}$-algebras, J. Funct. Anal. 13(1973), 277-301. MR0470685 (57:10431)

[2] B. Blackadar, K-Theory for Operator Algebras, MSRI Publication Series 5, Springer-Verlag, New York, Heidelberg, Berlin, Tokyo, 1986. MR0859867 (88g:46082)

[3] B. Blackadar, Matricial and ultramatricial topology, pages 11-38 in: Operator Algebras, Mathematical Physics and Low Dimensional Topology (Istanbul, 1991), R. Herman and B. Tanbay (eds.), A. K. Peters, Wellesley, MA, 1993. MR1259056 (95i:46102)

[4] B. Blackadar, Operator Algebras, Encyclopaedia of Mathematical Sciences vol. 122, SpringerVerlag, Berlin, 2006. MR2188261 (2006k:46082)

[5] B. Blackadar, M. Dădărlat, and M. Rørdam, The real rank of inductive limit $C^{*}$-algebras, Math. Scand. 69(1991), 211-216. MR1156427 (93e:46067)

[6] O. Bratteli, G. A. Elliott, D. E. Evans, and A. Kishimoto, Finite group actions on AF algebras obtained by folding the interval, K-Theory 8(1994), 443-464. MR1310287 (96f:46125)

[7] M. Dădărlat and G. Gong, A classification result for approximately homogeneous $C^{*}$-algebras of real rank zero, Geom. Funct. Anal. 7(1997), 646-711. MR1465599 (98j:46062)

[8] M. Dădărlat, G. Nagy, A. Némethi, and C. Pasnicu, Reduction of topological stable rank in inductive limits of $C^{*}$-algebras, Pacific J. Math. 153(1992), 267-276. MR.1151561 (93d:46119)

[9] J. Dixmier, $C^{*}$-Algebras, North-Holland, Amsterdam, New York, Oxford, 1977. MR0458185 (56:16388)

[10] P. Donovan and M. Karoubi, Graded Brauer groups and K-theory with local coefficients, Publ. Math. IHES 30(1970), 5-25. MR0282363 (43:8075)

[11] S. Eilenberg and N. E. Steenrod, Foundations of Algebraic Topology, Princeton University Press, Princeton, 1952. MR0050886 (14:398b)

[12] G. A. Elliott, On the classification of $C^{*}$-algebras of real rank zero, J. Reine Angew. Math. 443(1993), 179-219. MR 1241132 (94i:46074)

[13] R. Engelking, Dimension Theory, North-Holland, Oxford, Amsterdam, New York, 1978. MR 0482697 (58:2753b)

[14] J. M. G. Fell, The structure of algebras of operator fields, Acta Math. 106(1961), 233-280. MR0164248 (29:1547)

[15] K. Goodearl, Riesz decomposition in inductive limit $C^{*}$-algebras, Rocky Mtn. J. Math. 24(1994), 1405-1430. MR1322235 (96c:46053)

[16] D. Husemoller, Fibre Bundles (3rd ed.), Springer-Verlag, New York, Berlin, Heidelberg, London, Paris, Tokyo, Hong Kong, Barcelona, Budapest, 1994. MR1249482 (94k:55001)

[17] X. Jiang and $\mathrm{H}$. Su, A classification of simple limits of splitting interval algebras, J. Funct. Anal. 151(1997), 50-76. MR1487770 (99d:46076)

[18] X. Jiang and H. Su, On a simple unital projectionless $C^{*}$-algebra, Amer. J. Math. 121(1999), 359-413. MR1680321 (2000a:46104)

[19] J. L. Kelley, General Topology, Van Nostrand Reinhold, New York, Cincinnati, Toronto, London, Melbourne, 1955. MR0070144 (16:1136c)

[20] H. Lin and N. C. Phillips, Crossed products by minimal homeomorphisms, in preparation.

[21] Q. Lin, Analytic structure of the transformation group $C^{*}$-algebra associated with minimal dynamical systems, preprint. 
[22] Q. Lin and N. C. Phillips, Ordered K-theory for $C^{*}$-algebras of minimal homeomorphisms, pages 289-314 in: Operator Algebras and Operator Theory, L. Ge, et al. (eds.), Contemporary Mathematics vol. 228, 1998. MR 1667666 (2000a:46118)

[23] Q. Lin and N. C. Phillips, Direct limit decomposition for $C^{*}$-algebras of minimal diffeomorphisms, pages 107-133 in: Operator Algebras and Applications, Advanced Studies in Pure Mathematics vol. 38, Mathematical Society of Japan, 2004. MR2059804 (2005d:46144)

[24] Q. Lin and N. C. Phillips, The structure of $C^{*}$-algebras of minimal diffeomorphisms, in preparation.

[25] T. A. Loring, Lifting Solutions to Perturbing Problems in $C^{*}$-Algebras, Fields Institute Monographs no. 8, American Mathematical Society, Providence, RI, 1997. MR 1420863(98a:46090)

[26] M. Martin and C. Pasnicu, Some comparability results in inductive limit $C^{*}$-algebras, J. Operator Theory 30(1993), 137-147. MR.1302612 (96a:46109)

[27] J. Milnor, Differential Topology, mimeographed notes, Princeton University, 1958.

[28] J. R. Munkres, Topology: A First Course, Prentice-Hall, Englewood Cliffs, NJ, 1975. $\operatorname{MR} 0464128(57: 4063)$

[29] J. Mygind, Classification of simple inductive limits of interval algebras with dimension drops, preprint 1998.

[30] J. Mygind, Classification of certain simple $C^{*}$-algebras with torsion in $K_{1}$, Canad. J. Math. 53(2001), 1223-1308. MR1863849 (2002i:46054)

[31] A. R. Pears, Dimension Theory of General Spaces, Cambridge University Press, Cambridge, London, New York, Melbourne, 1975. MR0394604 (52:15405)

[32] G. K. Pedersen, Pullback and pushout constructions in $C^{*}$-algebra theory, J. Funct. Anal. 167(1999), 243-344. MR.1716199 (2000j:46105)

[33] N. C. Phillips, Representable K-theory for $\sigma$-C*-algebras, K-Theory 3(1989), 441-478. MR.1050490 (91k:46082)

[34] N. C. Phillips, The $C^{*}$ projective length of $n$-homogeneous $C^{*}$-algebras, J. Operator Theory 31(1994), 253-276. MR1331776 (96j:46058)

[35] N. C. Phillips, Cancellation and stable rank for direct limits of recursive subhomogeneous algebras, Trans. Amer. Math. Soc., this issue.

[36] N. C. Phillips, Real rank and property (SP) for direct limits of recursive subhomogeneous algebras, Trans. Amer. Math. Soc., to appear.

[37] M. A. Rieffel, $C^{*}$-algebras associated with irrational rotations, Pacific J. Math. 93(1981), 415-429. MR0623572 (83b:46087)

[38] M. A. Rieffel, Dimension and stable rank in the K-theory of $C^{*}$-algebras, Proc. London Math. Soc. Ser. 3 46(1983), 301-333. MR0693043 (84g:46085)

[39] M. A. Rieffel, The homotopy groups of the unitary groups of noncommutative tori, J. Operator Theory 17(1987), 237-254. MR0887221 (88f:22018)

[40] E. H. Spanier, Algebraic Topology, McGraw-Hill, New York, San Francisco, St. Louis, Toronto, London, Sydney, 1966. MR0210112 (35:1007)

[41] R. G. Swan, Vector bundles and projective modules, Trans. Amer. Math. Soc. 105(1962), 264-277. MR0143225 (26:785)

[42] K. Thomsen, Limits of certain subhomogeneous $C^{*}$-algebras, Mém. Soc. Math. Fr. (N.S.) No. 71(1997). MR1649315 (2000c:46110)

[43] J. Tomiyama and M. Takesaki, Applications of fibre bundles to the certain class of $C^{*}$ algebras, Tôhoku Math. J. (2) 13(1961), 498-522. MR0139025(25:2465)

[44] N. B. Vasil'ev, $C^{*}$-algebras with finite-dimensional irreducible representations, Uspehi Mat. Nauk 21(1966), no. 1, 135-154 (in Russian); English translation in: Russian Math. Surveys 21(1966), no. 1, 137-155. MR0201994(34:1871)

Department of Mathematics, University of Oregon, Eugene, Oregon 97403-1222 